\title{
Stochastic Approximation for Consensus: A New Approach via Ergodic Backward Products
}

\author{
Minyi Huang, Member, IEEE
}

\begin{abstract}
This paper considers both synchronous and asynchronous consensus algorithms with noisy measurements. For stochastic approximation based consensus algorithms, the existing convergence analysis with dynamic topologies heavily relies on quadratic Lyapunov functions, whose existence, however, may be difficult to ensure for switching directed graphs. Our main contribution is to introduce a new analytical approach. We first show a fundamental role of ergodic backward products for mean square consensus in algorithms with additive noise. Subsequently, we develop ergodicity results for backward products of degenerating stochastic matrices converging to a $0-1$ matrix via a discrete time dynamical system approach. These results complement the existing ergodic theory of stochastic matrices and provide an effective tool for analyzing consensus problems. Under a joint connectivity assumption, our approach may deal with switching topologies, delayed measurements and correlated noises, and it does not require the double stochasticity condition typically assumed for the existence of quadratic Lyapunov functions.
\end{abstract}

Index Terms-Backward product, consensus, delay, ergodicity, mean square convergence, measurement noise, stochastic approximation, synchronous and asynchronous algorithms.

\section{INTRODUCTION}

D URING the past decade, an enormous amount of research effort has been devoted to consensus problems and various closely related formulations for multi-agent systems [8], [16], [20], [23], [32], [36]. A comprehensive survey may be found in [31], [37]. In recent years, consensus algorithms with imperfect information exchange have attracted significant attention, addressing additive noise [2], [3], [12], [30], [34], [38], [39], [46], [47] or quantization effect [4], [10], [11], [22]. The classic work [44] considered consensus algorithms for distributed function optimization with noisy gradient information. Models with noisy measurements take into account random uncertainty in signal reception and characterize more realistic network conditions. Also, when probabilistic quantizers are used to eliminate bias, quantization errors may be modeled as additive noise of zero mean [4].

For consensus models with noisy measurements, stochastic approximation with a decreasing step size may be applied such

Manuscript received May 07, 2010; revised January 19, 2011, January 20, 2011, and September 19, 2011; accepted April 03, 2012. Date of publication May 14, 2012; date of current version November 21, 2012. This work was supported in part by a Discovery Grant of the Natural Sciences and Engineering Research Council (NSERC) of Canada. A preliminary version of this paper was presented at the 49th IEEE CDC Conference, 2010. Recommended by Associate Editor C. Szepesvari.

The author is with the School of Mathematics and Statistics, Carleton University, Ottawa, ON K1S 5B6, Canada (e-mail: mhuang@math.carleton.ca).

Digital Object Identifier 10.1109/TAC.2012.2199149 that each agent can gradually reduce the weights to its neighbors and hence attenuate the noise [18], [21], [35], [41]. A typical algorithm takes the form

$$
X_{t+1}=\left(I+a_{t} B_{t}\right) X_{t}+a_{t} D_{t} W_{t}, \quad t \geq 0
$$

where $X_{t}$ is a vector consisting of the states of $n$ nodes; $B_{t}=$ $\left(b_{i j}(t)\right)_{1 \leq i, j \leq n}$ is a matrix with zero row sums and nonnegative off-diagonal entries; $a_{t}>0$ is the step size; and $W_{t}$ is a noise vector with coefficient matrix $D_{t}$. For $i \neq j, b_{i j}(t)$ is nonzero if and only if there is an edge from node $j$ to node $i$ at time $t$.

For fixed network topologies, $B_{t}$ may be selected as a constant matrix $B$, and mean square and probability one convergence of the algorithm may be proved by either quadratic Lyapunov functions [18], [27] or change of coordinates [19].

Convergence analysis in switching networks has heavily relied on quadratic Lyaponuv functions [3], [17], [21], [28], [42], and an assumption often used is that the weight matrix $I+$ $a_{t} B_{t}$ is doubly stochastic for each $t$ (or starting from some $t_{0}$ ). The double stochasticity assumption was initially introduced for noiseless average-consensus problems to make the state average invariant [32]. For algorithm (1), this assumption ensures that the squared norm of the disagreement vector [28], [32] becomes a quadratic Lyapunov function. However, it is also very restrictive [5] and brings about serious feasibility issues in directed networks. To elucidate this aspect, let the $n \times n$ nonnegative matrix $B_{t}^{o}$ be the same as $B_{t}$ except for all zero diagonal entries. We call $B_{t}^{o}$ a weight balanced matrix if the $i$ th row sum equals the $i$ th column sum for each $i$. Suppose that $I+a_{t} B_{t}$ is a nonnegative matrix and $a_{t}>0$; then obviously $I+a_{t} B_{t}$ is doubly stochastic if and only if $B_{t}^{o}$ is weight balanced, and it is known that such a matrix $B_{t}^{o}$ exists if and only if the directed graph modeling the network is strongly semiconnected [15] (i.e., if there exists a directed path from node $i$ to node $j$, then there exists one from $j$ to $i$ ). In randomly varying directed networks, it is demanding to maintain strong semiconnectedness, and so one cannot in general expect the existence of doubly stochastic weight matrices at all times.

In addition to the above nonexistence issue, the double stochasticity requirement also imposes significant computational difficulties. For undirected graphs, doubly stochastic weight matrices may be constructed online via Metropolis weights [46]. However, on time-varying directed graphs it is generally infeasible to construct such matrices online without global instantaneous network topology information even if they exist. Although distributed iterative algorithms are available for constructing doubly stochastic matrices over directed graphs [15], they are not applicable for dynamic topologies. So, it is 
of practical importance to study models using general weight matrices without double stochasticity.

The recent work [3] considers the consensus algorithm

$$
X_{t+1}=A_{t} X_{t}+F_{t} W_{t}, \quad t \geq 0
$$

where $A_{t}$ is a random matrix of unit row sums and $W_{t}$ is the additive noise. This model has considerable generality with respect to the past research; in particular, $A_{t}$ is not required to be nonnegative. The authors succeeded in developing Lyapunov analysis without double stochasticity conditions. They proved probability one convergence of the algorithm and estimated the convergence rate. Let $J=(1 / n) \mathbf{1}_{n \times n}$, where $\mathbf{1}_{n \times n}$ is an $n \times n$ matrix of all ones. The convergence proof in [3] uses $\left|X_{t}-J X_{t}\right|^{2}$ as a stochastic Lyapunov function and requires i) $\lambda_{1}\left\{E\left[A_{t}^{T}(I-J) A_{t}\right]\right\} \leq 1$, where $\lambda_{1}(\cdot)$ denotes the largest eigenvalue of a symmetric matrix, and ii) $\sum_{t=0}^{\infty}\left(1-\lambda_{1}\left\{E\left[A_{t}^{T}(I-J) A_{t}\right]\right\}\right)=\infty$, in addition to assumptions on the noise term. Although this approach achieves considerable modeling generality, the eigenvalue conditions i) and ii) are quite restrictive for application to stochastic approximation as illustrated by the following example.

Example: Let $\left\{A_{t}, t \geq 0\right\}$ be a deterministic sequence. Each $A_{t}$ takes a value from $\left\{I+a_{t} B^{(1)}, I+a_{t} B^{(2)}\right\}$, where $a_{t}=$ $1 /\left(50+t^{\gamma}\right)$ for some $\gamma \in(1 / 2,1]$ and

$$
B^{(1)}=\left[\begin{array}{ccc}
-24 & 0 & 24 \\
1 & -1 & 0 \\
0 & 1 & -1
\end{array}\right], \quad B^{(2)}=\left[\begin{array}{ccc}
-48 & 0 & 48 \\
1 & -2 & 1 \\
0 & 2 & -2
\end{array}\right]
$$

The sequence $\left\{W_{t}, t \geq 0\right\}$ consists of independent random vectors, $E W_{t}=0, \sup _{t \geq 0} E\left|W_{t}\right|^{2}<\infty, E\left|X_{0}\right|^{2}<\infty$, and $F_{t}=a_{t} I$.

By the structure of $B^{(1)}$ and $B^{(2)}$, the network underlying algorithm (2) is always strongly connected. However, the criteria in [3] cannot be used to assert convergence. Denote the characteristic polynomial $f^{(k)}(\lambda)=\operatorname{det}\left[\lambda I-\left(I+a_{t} B^{(k)}\right)^{T}(I-\right.$ $\left.J)\left(I+a_{t} B^{(k)}\right)\right], k=1,2$. Then $\lim _{\lambda \rightarrow \infty} f^{(k)}(\lambda)=\infty$, and by direct calculations, $f^{(1)}(1)=-(88 / 3) a_{t}^{2}+O\left(a_{t}^{3}\right), f^{(2)}(1)=$ $-(172 / 3) a_{t}^{2}+O\left(a_{t}^{3}\right)$. For sufficiently small $a_{t}, f^{(k)}(\lambda)=$ 0 has a root in $(1, \infty)$ by the intermediate value theorem; so $\lambda_{1}\left[A_{t}^{T}(I-J) A_{t}\right]>1$ and conditions i)-ii) are violated.

To overcome the inherent limitations of the existing methods, we develop a new approach to analyze stochastic approximation for consensus, and it will cover many practical models and the above example as well. In a very general setup of noisy consensus algorithms, we show that ergodicity of the backward products of the weight matrices is a necessary and sufficient condition for mean square consensus, which reveals the fundamental mechanism governing the convergent algorithms studied by different authors [3], [17], [21], [28], [42]. By checking ergodicity of backward products in switching models, one may be saved from the challenging task of searching for Lyapunov functions. For nonexistence of quadratic Lyapunov functions with general weight matrices, see [33].
The weight matrices in our stochastic approximation algorithm, starting with an appropriate initial time to ensure nonnegative matrices, form a sequence of stochastic matrices converging to a $0-1$ matrix, which will be called degenerating stochastic matrices due to this convergence feature. We note that there has existed an extensive literature (see [40] and references therein) on ergodicity of backward products of stochastic matrices. In particular, for analyzing inhomogeneous backward products, Wolfowitz's ergodicity theorem [20], [36], [45] and paracontraction [13], [25], [26] are well known powerful tools. Also, for the iterations of a nonnegative matrix with stationary delays, multiplicative ergodicity was studied via Lyaponov exponents in [14]. However, these results are not applicable to our model. To our best knowledge, this paper is the first to systematically establish ergodic theorems for backward products of degenerating stochastic matrices. We introduce a key notion of compatible nonnegative matrices and develop a dynamical system approach to prove ergodicity, which differs from the classical approaches in [40], [45].

Our approach to analyze the stochastic approximation algorithm differs from the Lyapunov approach by a shift of attention from ensuring steady energy decay to ergodicity check. This strategy makes it possible to overcome the weakness of the existing methods. Also, we note that this paper only addresses mean square convergence of the stochastic approximation algorithm. The further analysis of its sample path behavior is an interesting problem.

The main contributions of the paper are summarized as follows:

- We show a fundamental role of ergodic backward products for mean square consensus, and establish ergodic theorems for degenerating stochastic matrices.

- The ergodicity approach is applied to prove mean square consensus for a large class of models which the existing Lyapunov approaches cannot handle; specifically, it enables us to treat general weight matrices over switching networks, delayed noisy measurements, synchronous and asynchronous algorithms, and also correlated noises.

The organization of the paper is as follows. Section II formulates the stochastic consensus problem. Section III shows a necessary and sufficient condition for mean square consensus via ergodic backward products. Section IV introduces compatible nonnegative matrices and Section V proves ergodicity of degenerating stochastic matrices. Section VI shows mean square consensus, and Section VII concludes the paper.

We make some convention about notation. The node index is often used as a superscript in different variables $\left(x_{t}^{i}, z_{t}^{i}\right.$, etc.) and should not be interpreted as an exponent of a number. For a matrix $M$, the element in the $i$ th row and the $j$ th column is called the $(i, j)$ th element and denoted by $M(i, j)$. For a vector or matrix $M$, denote the Frobenius norm $|M|=\left[\operatorname{Tr}\left(M^{T} M\right)\right]^{1 / 2}$. Let $\mathbf{1}_{k} \in \mathbb{R}^{k}$ denote a column vector of $k$ ones. For column vectors $Z_{1}, \ldots, Z_{l},\left[Z_{1} ; \ldots ; Z_{l}\right]$ denotes the column vector obtained by vertical concatenation of the $l$ vectors. For two sets $S_{1}$ and $S_{2}$, the set $S_{1} \backslash S_{2}$ consists of all elements which are in $S_{1}$ but not in $S_{2}$. The abbreviation w.p.1 stands for "with probability one." We use $C$ (or $C_{0}, C_{1}, \ldots$ ) to denote a generic positive constant which may vary at different places. 


\section{Stochastic CONSEnsus Algorithm}

For the reader's convenience, we provide a list of key notation used in the analysis. ${ }^{1}$

$G \quad$ Digraph with nodes $\mathcal{N}=\{1, \ldots, n\}$ and edges $\mathcal{E}$.

$A_{G} \quad$ Adjacency matrix of $G$

$G_{t} \quad$ Random digraph with nodes $\mathcal{N}$ and edges $\mathcal{E}_{t}$.

$G_{\left[t_{1}, t_{2}\right)} \quad$ Union of the digraphs $G_{t}, t_{1} \leq t<t_{2}$.

$x_{t}^{i} \quad$ State of node $i$.

$y_{t}^{i k}$

$w_{t}^{i k}$

$d_{t}^{i k}$

$d^{*}$

$d_{1}^{*}$

$\theta_{t}$

$a_{t}$

$b_{i k}(t)$

$B_{t}$

$\mathbf{B}_{t}$

$\mathbf{U}$

$\mathbf{D}_{t}$

$X_{t}, X_{t}^{(-d)}$

$\mathbf{X}_{t}$

$W_{t}$

$Z_{t}$

Noisy measurement node $i$ obtains from node $k$.

Additive noise in $y_{t}^{i k}$.

Measurement delay along edge $(k, i)$.

Deterministic upper bound of all $d_{t}^{i k}$.

$d^{*}+1$.

Counters $\left(\theta_{t}^{1}, \ldots, \theta_{t}^{n}\right)$ of $n$ nodes.

Step size for state update.

Weight parameter node $i$ assigns to node $k$.

$n \times n$ matrix $\left(b_{i k}(t)\right)_{1 \leq i, k \leq n}$ of zero row sums. $n d_{1}^{*} \times n d_{1}^{*}$ matrix.

$n d_{1}^{*} \times n d_{1}^{*}$ stochastic matrix of $0-1$ elements.

$n d_{1}^{*} \times n_{1}$ noise coefficient matrix.

$n$-dimensional vector.

$n d_{1}^{*}$-dimensional vector.

$n_{1}$-dimensional noise vector.

tries in $X_{t}$.

$\mathbf{A}_{t} \quad n d_{1}^{*} \times n d_{1}^{*}$ stochastic matrix.

$M_{t}, M_{t}^{A} \quad$ Stochastic matrices.

$\Phi_{t, s}, M^{t, s} \quad$ Backward product of stochastic matrices.

We introduce some standard preliminaries on graph modeling of the network topology. A directed graph (or digraph) $G=$ $(\mathcal{N}, \mathcal{E})$ consists of a set of nodes $\mathcal{N}=\{1, \ldots, n\}$ and a set of directed edges $\mathcal{E}$. A directed edge (simply called an edge) is denoted by an ordered pair $(i, j) \in \mathcal{N} \times \mathcal{N}$, where $i \neq j$. A directed path from node $i_{1}$ to node $i_{l}$ consists of a sequence of nodes $i_{1}, \ldots, i_{l}, l \geq 2$, such that $\left(i_{k}, i_{k+1}\right) \in \mathcal{E}$ for all $k \leq$ $l-1$. The digraph $G$ is strongly connected if from any node to any other node, there exists a directed path. A directed tree is a digraph where each node $i$, except the root, has exactly one parent node $j$ so that $(j, i) \in \mathcal{E}$. We call $G^{\prime}=\left(\mathcal{N}^{\prime}, \mathcal{E}^{\prime}\right)$ a subgraph of $G$ if $\mathcal{N}^{\prime} \subset \mathcal{N}$ and $\mathcal{E}^{\prime} \subset \mathcal{E}$. The digraph $G$ is said to contain a spanning tree if there exists a directed tree $G_{\mathrm{tr}}=\left(\mathcal{N}, \mathcal{E}_{\mathrm{tr}}\right)$ as a subgraph of $G$. The adjacency matrix of $G$ is an $n \times n$ matrix $A_{G}=\left(a_{i j}\right)_{1 \leq i, j \leq n}$, where $a_{i j}=1$ if $(i, j) \in \mathcal{E}$, and $a_{i j}=0$ otherwise.

The dynamic network topology to specify the signal reception is modeled by a sequence of digraphs $\left\{G_{t}=\left(\mathcal{N}, \mathcal{E}_{t}\right), t \geq 0\right\}$, where $\mathcal{N}=\{1, \ldots, n\}$ and $\mathcal{E}_{t}$ randomly changes with time. We may view $\left\{\mathcal{E}_{t}, t \geq 0\right\}$ as a set-valued random process. The

\footnotetext{
${ }^{1}$ Letters in boldface usually denote vectors or matrices built upon more basic ones in lower dimensions.
}

adjacency matrix $A_{G_{t}}$ is a matrix-valued random variable. So $\mathcal{E}_{t}$ is completely determined by $A_{G_{t}}$. If $(j, i) \in \mathcal{E}_{t}$, node $i$ receives information from node $j$ which is called a neighbor of node $i$. The neighbor set of node $i$ is denoted by $\mathcal{N}_{i, t}=\left\{j \mid(j, i) \in \mathcal{E}_{t}\right\}$.

\section{A. Stochastic Approximation Algorithm}

Let the underlying probability space be denoted by $(\Omega, \mathcal{F}, P)$, corresponding to the sample space, the collection of all events, and the probability measure, respectively. At time $t \in\{0,1,2, \ldots\}$, node $i$ is associated with a real-valued state $x_{t}^{i}$. Each node knows its own state exactly. Define the state vector

$$
X_{t}=\left[x_{t}^{1}, \ldots, x_{t}^{n}\right]^{T}, \quad t \geq 0
$$

The initial state vector is $X_{0}$. At time $t$, if $\mathcal{N}_{i, t} \neq \emptyset$ (the empty set), node $i$ receives possibly outdated information from its neighbors, which is modeled by

$$
y_{t}^{i k}=x_{t-d_{t}^{i k}}^{k}+w_{t}^{i k}, \quad k \in \mathcal{N}_{i, t}
$$

where $w_{t}^{i k}$ is the noise and $d_{t}^{i k} \geq 0$ is an integer-valued random delay. Since the system starts at $t=0$, the implicit requirement for the neighbor set is that

$$
k \in \mathcal{N}_{i, t} \quad \text { implies } \quad t-d_{t}^{i k} \geq 0 \text {. }
$$

A fixed upper bound for $d_{t}^{i k}$ will be specified later. Each node will use its own state and its noisy measurements to form a weighted average.

According to the local information exchange, we define the matrix $B_{t}=\left(b_{i k}(t)\right)_{1 \leq i, k \leq n}$ as follows. If $\mathcal{N}_{i, t}=\emptyset$, define

$$
b_{i k}(t)=0 \quad \text { for all } k \in \mathcal{N} .
$$

If $\mathcal{N}_{i, t} \neq \emptyset$, set

$$
\begin{cases}b_{i k}(t) \in[\underline{b}, \bar{b}], & \text { if } k \in \mathcal{N}_{i, t} \\ b_{i k}(t)=0, & \text { if } k \notin \mathcal{N}_{i, t} \cup\{i\} \\ b_{i i}(t)=-\sum_{k \in \mathcal{N}_{i, t}} b_{i k}(t) & \end{cases}
$$

where $0<\underline{b} \leq \bar{b}<\infty$ are two deterministic constants. We may interpret $B(t)$ as the generator of a continuous time Markov chain with $n$ states. The specification (5), (6) is a generalization of the weights with a fixed network [19] to randomly varying networks, and $\left\{B_{t}, t \geq 0\right\}$ is a matrix-valued random process.

Each node maintains a counter $\theta_{t}^{i}$. Denote $\theta_{t}=\left[\theta_{t}^{1}, \ldots, \theta_{t}^{n}\right]$. We describe two cases.

(SU) Synchronous update:

$$
\theta_{t}^{i}=t, \quad i \in \mathcal{N}, \quad t \geq 0
$$

where the nodes need to share slotted time.

(AU) Asynchronous update:

$$
\theta_{t}^{i}=\sum_{s=1}^{t} 1_{\left\{\left|\mathcal{N}_{i, s}\right|>0\right\}}, \quad i \in \mathcal{N}, \quad t \geq 1
$$

and $\theta_{0}^{i}=0$, where $\left|\mathcal{N}_{i, s}\right|$ is the number of neighbors of node $i$ at time $s$. So (8) means that the node increases its counter by one whenever it receives signals from its neighbors. 
The consensus algorithms for cases (SU) and (AU) are specified in a unified manner. At time $t \geq 0$, if $\mathcal{N}_{i, t}=\emptyset$, set $x_{t+1}^{i} \equiv x_{t}^{i}$. If $\mathcal{N}_{i, t} \neq \emptyset$, node $i$ updates its state by the rule

$$
x_{t+1}^{i}=\left[1+a_{\theta_{t}^{i}} b_{i i}(t)\right] x_{t}^{i}+a_{\theta_{t}^{i}} \sum_{k \in \mathcal{N}_{i, t}} b_{i k}(t) y_{t}^{i k}, \quad t \geq 0
$$

where $\left\{a_{t}, t \geq 0\right\}$ is a sequence of positive step sizes. We call $I+\operatorname{Diag}\left(a_{\theta_{t}^{1}}, \ldots, a_{\theta_{t}^{n}}\right) B_{t}$ the weight matrix.

When the step size is updated using (8), the resulting asynchronous algorithm (9) is essentially driven by event times, i.e., the moments of receiving signals. Once initialized, the algorithm may be implemented without synchronized time slots although we use the pre-specified discrete times $0,1,2, \ldots$ to describe (9). For related literature on asynchronous stochastic approximation, see [1], [7], [24], and [43].

The actually observed network topology at time $t$ may be denoted by $G_{t}(\omega)=\left(\mathcal{N}, \mathcal{E}_{t}(\omega)\right)$ to indicate its dependence on the sample. Denote the maximal set of communication links $\mathcal{E}_{\max }=\left\{(k, i) \mid P\left((k, i) \in \mathcal{E}_{t}\right)>0\right.$ for some $\left.t \geq 0\right\}$. For convenience of statistical modeling of the noises and delay, we make the convention: $w_{t}^{i k}$ and $d_{t}^{i k}$ are defined for all $(k, i) \in$ $\mathcal{E}_{\max }$. If $(k, i)$ does not appear in $\mathcal{E}_{t}$ so that the measurement relation (3) does not physically occur, we still introduce $w_{t}^{i k}$ and $d_{t}^{i k}$ as dummy random variables. Let the random variables $\left\{w_{t}^{i k} \mid(k, i) \in \mathcal{E}_{\max }\right\}$ be listed by a fixed ordering of $(k, i)$ to obtain a noise vector $W_{t}$ of $n_{1}$ dimension.

Definition 1: The $n$ nodes are said to achieve mean square consensus if $E\left|x_{t}^{i}\right|^{2}<\infty, t \geq 0,1 \leq i \leq n$, and there exists a random variable $x^{*}$ such that $\lim _{t \rightarrow \infty} E\left|x_{t}^{i}-x^{*}\right|^{2}=0$ for $1 \leq i \leq n$.

There have existed some effective approaches to analyze asynchronous stochastic approximation and show probability one convergence (see, e.g., [1], [43]). These algorithms are typically associated with an underlying time-invariant mapping, and the contraction property of the mapping may be exploited [43] or the ordinary differential equation (ODE) approach may be used after appropriate scaling of time [1] where the asynchronous algorithm behaves like a synchronous one with small perturbations. To analyze our model, due to the rapid switches of $B_{t}$, it is difficult to apply these approaches and it is necessary to develop a different method.

\section{B. Main Assumptions}

(A1) The deterministic sequence $\left\{a_{t}, t \geq 0\right\}$ satisfies

$$
a_{0}>0, \quad \text { and } \quad \alpha t^{-\gamma} \leq a_{t} \leq \beta t^{-\gamma} \text { for } t \geq 1
$$

where $\gamma \in(1 / 2,1]$ and $0<\alpha \leq \beta<\infty$.

So (A1) implies the standard step size condition used in stochastic approximation: $\sum_{t=0}^{\infty} a_{t}=\infty$ and $\sum_{t=0}^{\infty} a_{t}^{2}<\infty$.

For two integers $0 \leq t_{1}<t_{2}$, define the digraph $G_{\left[t_{1}, t_{2}\right)}=$ $\left(\mathcal{N}, \cup_{t_{1} \leq t<t_{2}} \mathcal{E}_{t}\right)$, which is called the union of the collection of digraphs $\left\{G_{t} \mid t_{1} \leq t<t_{2}\right\}$. Since the sequence $\left\{G_{t}, t \geq 0\right\}$ depends on the sample $\omega, G_{\left[t_{1}, t_{2}\right)}$ also depends on $\omega$. We introduce the following assumption.

(A2) There exists an infinite sequence of integer-valued random variables $0 \equiv T_{0}<T_{1}<T_{2}<\ldots$ such that the two conditions hold:
1) $G_{\left[T_{l}, T_{l+1}\right)}$ is strongly connected w.p. 1 for $l \geq 0$;

2) $\alpha_{1}:=\sup _{l \geq 1}\left(T_{l}-T_{l-1}\right)<\infty$ w.p.1.

It will be helpful to provide some explanation on notation. Note that given a sample $\omega \in \Omega, G_{t}(\omega)$ has the set of edges $\mathcal{E}_{t}(\omega)$. The random digraph $G_{\left[T_{l}, T_{l+1}\right)}$ has the set of nodes $\mathcal{N}$. Given $\omega, G_{\left[T_{l}, T_{l+1}\right)}$ is interpreted as $G_{\left[T_{l}(\omega), T_{l+1}(\omega)\right)}(\omega)$ possessing the set of edges $\cup_{T_{l}(\omega) \leq t<T_{l+1}(\omega)} \mathcal{E}_{t}(\omega)$.

(A3) $\left\{W_{t}, t \geq 0\right\}$ is a sequence of independent random vectors of zero mean and is independent of $\left\{\left(B_{t}, A_{G_{t}},\left\{d_{t}^{i k} \mid(k, i) \in \mathcal{E}_{\max }\right\}\right), t \geq 0\right\}$, where $0 \leq d_{t}^{i k} \leq d^{*}$ w.p.1 for a fixed integer $d^{*}$. In addition, $E\left|X_{0}\right|^{2}<\infty$ and $\sup _{t>0} E\left|W_{t}\right|^{2}<\infty$.

The delay upper bound $d^{*}$ is used for analyzing the stochastic approximation algorithm, which may be implemented without knowing the value of $d^{*}$. To deal with leader following, we introduce another type of connectivity condition.

$\left(\mathbf{A} 2^{\prime}\right)$ There is a fixed leader node $i_{L}$ which has no neighbor in each $G_{t}$. There exists an infinite sequence of integervalued random variables $0 \equiv T_{0}<T_{1}<T_{2}<\ldots$ such that w.p.1, $G_{\left[T_{l}, T_{l+1}\right)}$ contains a spanning tree with root $i_{L}$ for $l \geq 0$. In addition, (A2)-2) is satisfied.

Under $\left(\mathbf{A} \mathbf{2}^{\prime}\right)$, there is no edge from other nodes to node $i_{L}$, so that $B_{t}$ defined by (5), (6) necessarily has all zero elements on the $i_{L}$ th row.

\section{Vector Form of the Algorithm}

For $t \geq 0$, denote the set of $n \times n$ random matrices

$$
B_{d, t}=\left(B_{d, t}(i, k)\right)_{1 \leq i, k \leq n}, \quad d=0,1, \ldots, d^{*} .
$$

For their diagonal elements, we take $B_{0, t}(i, i)=b_{i i}(t)$ and $B_{d, t}(i, i)=0, d=1, \ldots, d^{*}$, for all $i$. For $d=0,1, \ldots, d^{*}$, the off-diagonal element $B_{d, t}(i, k)$ is nonzero and further taken as $b_{i k}(t)$ if and only if $b_{i k}(t)>0$ and $d_{t}^{i k}=d$. Denote

$$
\mathbf{a}_{\theta_{t}}^{0}=\operatorname{Diag}\left(a_{\theta_{t}^{1}}, \ldots, a_{\theta_{t}^{n}}\right), \quad \mathbf{B}_{t}^{0}=\left[B_{0, t}, B_{1, t}, \ldots, B_{d^{*}, t}\right] .
$$

The $i$ th row of $\mathbf{B}_{t}^{0}$ contains the same set of nonzero elements as the $i$ th row of $B_{t}$ does. Due to (4), if $t<d^{*}$, we necessarily have $B_{d, t}=0$ for all $d=t+1, \ldots, d^{*}$.

We write (9) in the equivalent form

$$
X_{t+1}=X_{t}+\mathbf{a}_{\theta_{t}}^{0} \mathbf{B}_{t}^{0}\left[X_{t} ; X_{t-1} ; \ldots ; X_{t-d^{*}}\right]+\mathbf{a}_{\theta_{t}}^{0} D_{t} W_{t}, \quad t \geq 0
$$

where $D_{t}$ is an $n \times n_{1}$ random matrix determined by $B_{t}$ and we set $X_{t} \equiv 0$ for $-d^{*} \leq t<0$. If $d^{*}=0$ and all nodes update their step sizes by (7), then (12) reduces to (1).

For $d_{1}^{*}=d^{*}+1$, denote the $n d_{1}^{*} \times n d_{1}^{*}$ matrix

$$
\mathbf{U}=\left[\begin{array}{ccccc}
I & 0 & 0 & \cdots & 0 \\
I & 0 & 0 & \cdots & 0 \\
0 & I & 0 & \cdots & 0 \\
\vdots & \vdots & \ddots & \vdots & \vdots \\
0 & 0 & \ldots & I & 0
\end{array}\right]
$$

where each identity matrix is $n \times n$, and denote $\mathbf{a}_{\theta_{t}}=$ $\operatorname{Diag}\left(\mathbf{a}_{\theta_{t}}^{0}, 0_{n d^{*} \times n d^{*}}\right)$,

$$
\mathbf{B}_{t}=\left[\begin{array}{c}
\mathbf{B}_{\mathbf{t}}^{\mathbf{0}} \\
0_{n d^{*} \times n d_{1}^{*}}^{*}
\end{array}\right], \quad \mathbf{D}_{t}=\left[\begin{array}{c}
D_{t} \\
0_{n d^{*} \times n_{1}}
\end{array}\right] .
$$


It is clear that $\mathbf{B}_{t}$ is determined by $\left(B_{t},\left\{d_{t}^{i k} \mid(k, i) \in \mathcal{E}_{\max }\right\}\right)$.

Denote $\mathbf{X}_{t}=\left[X_{t} ; X_{t-1} ; \ldots ; X_{t-d^{*}}\right] \in \mathbb{R}^{n d_{1}^{*}}$. We have the state space representation

$$
\mathbf{X}_{t+1}=\left(\mathbf{U}+\mathbf{a}_{\theta_{t}} \mathbf{B}_{t}\right) \mathbf{X}_{t}+\mathbf{a}_{\theta_{t}} \mathbf{D}_{t} W_{t}, \quad t \geq 0
$$

where $X_{t} \equiv 0$ for $-d^{*} \leq t<0$. If all counters increase to infinity, there exists $t_{0}(\omega)$, depending on the sample $\omega$, such that $\left\{\mathbf{U}+\mathbf{a}_{\theta_{t}(\omega)} \mathbf{B}_{t}(\omega), t \geq t_{0}(\omega)\right\}$ is a sequence of stochastic matrices converging to the $0-1$ stochastic matrix $\mathbf{U}$. In this case, $\left\{\mathbf{U}+\mathbf{a}_{\theta_{t}(\omega)} \mathbf{B}_{t}(\omega), t \geq t_{0}(\omega)\right\}$ is called a sequence of degenerating stochastic matrices.

\section{NECESSARY AND SUFFICIENT CONDITION FOR CONSENSUS}

We use a general algorithm to reveal a fundamental relationship between mean square consensus and ergodicity of backward matrix products. Consider the system

$$
Y_{t+1}=A_{t} Y_{t}+H_{t} V_{t}, \quad t \geq 0
$$

where $Y_{t} \in \mathbb{R}^{m_{1}}$ denotes the states of $m_{1}$ agents, $V_{t} \in \mathbb{R}^{m_{2}}$ is the noise vector, and the initial condition is $Y_{0}$. Here $\left\{A_{t}, t \geq\right.$ $0\}$ and $\left\{H_{t}, t \geq 0\right\}$ are two sequences of random matrices of compatible dimensions. For each fixed $\omega, A_{t}(\omega)$ is a stochastic matrix for all $t \geq 0$. The model (16) includes (15) as a special case when the coefficient matrices of $\mathbf{X}_{t}$ in (15) are nonnegative for all $t \geq 0$.

\section{A. Ergodicity of Backward Products}

Let $\left\{\tilde{A}_{t}, t \geq 0\right\}$ be a sequence of deterministic nonnegative matrices, where each $\tilde{A}_{t}$ is a stochastic matrix. Define the so-called backward product

$$
\Phi_{t, s}=\tilde{A}_{t-1} \ldots \tilde{A}_{s} \quad \text { for } t>s \geq 0, \quad \Phi_{s, s}:=I .
$$

The product $\Phi_{t, s}$ is still a stochastic matrix. Let $\Phi_{t, s}(i, j)$ denote its $(i, j)$ th element.

Definition 2: [40] We say weak ergodicity holds for backward products of the sequence of stochastic matrices $\left\{\tilde{A}_{t}, t \geq\right.$ $0\}$ if

$$
\lim _{t \rightarrow \infty}\left|\Phi_{t, s}\left(i_{1}, j\right)-\Phi_{t, s}\left(i_{2}, j\right)\right|=0
$$

for any given $s \geq 0$ and $i_{1}, i_{2}, j$, i.e., the difference between any two rows of $\Phi_{t, s}$ converges to zero as $t \rightarrow \infty$. If in addition to weak ergodicity, $\Phi_{t, s}(i, j)$ converges as $t \rightarrow \infty$, for any $s, i, j$, we say strong ergodicity holds.

By [40, p. 154, Th. 4.17], weak and strong ergodicity are equivalent for backward products of any sequences of stochastic matrices. Hence, in the following we only speak of ergodicity of backward products.

It is worth mentioning that weak and strong ergodicity may also be defined for forward products of the form $\Phi_{s, t}^{f}=\tilde{A}_{s} \ldots \tilde{A}_{t-1}$ for $t>s$, and $\Phi_{s, s}^{f}:=I$, and that weak ergodicity differs from strong ergodicity. For an example showing divergence of the forward products of weakly ergodic stochastic matrices, see [9, p. 240].

\section{B. Necessary and Sufficient Condition for Consensus}

For the theorem below, we run algorithm (16) with any initial time-state pair $\left(t_{0}, Y_{t_{0}}\right)$. Denote $Y_{t}=\left[Y_{t}^{1}, \ldots, Y_{t}^{m_{1}}\right]^{T}, \Psi_{t, s}=$ $A_{t-1} \ldots A_{s}$ for $t>s$, and $\Psi_{s, s}:=I$.

Theorem 3: Assume

i) $\left\{V_{t}, t \geq 0\right\}$ is a sequence of random vectors of zero mean, independent of $\left\{\left(A_{t}, H_{t}\right), t \geq 0\right\}$;

ii) $\sum_{t=0}^{\infty} E\left|H_{t}\right|^{2} E\left|V_{t}\right|^{2}<\infty$;

iii) there exists a sequence of nonnegative numbers $\left\{\phi_{k}, k \geq\right.$ $0\}$ such that

$$
\left|E\left[V_{k} V_{k^{\prime}}^{T}\right]\right| \leq \phi_{\left|k-k^{\prime}\right|}\left(E\left|V_{k}\right|^{2} E\left|V_{k^{\prime}}\right|^{2}\right)^{\frac{1}{2}}, \quad \sum_{k=0}^{\infty} \phi_{k}<\infty .
$$

Then (16) ensures mean square consensus for any initial timestate pair $\left(t_{0}, Y_{t_{0}}\right)$ with $E\left|Y_{t_{0}}\right|^{2}<\infty$ if and only if $\left\{A_{t}, t \geq 0\right\}$ has ergodic backward products w.p.1.

Remark: If the random vectors $\left\{V_{t}, t \geq 0\right\}$ are independent with $E V_{t}=0$ and $E\left|V_{t}\right|^{2}<\infty$, iii) holds with $\phi_{0}=1$ and $\phi_{k}=0$ for all $k>0$.

The proof of Theorem 3 is given in Appendix A. In the context of stochastic approximation for consensus seeking, condition ii) in Theorem 3 is easy to satisfy since a decreasing step size may be used to attenuate the measurement noise. Thus, to a very large extent, the analysis of the asymptotic behavior of consensus algorithms of the form (16) reduces to checking the ergodicity condition along sample paths.

\section{Compatible Nonnegative Matrices}

This section develops some basic tools for analyzing sequences of degenerating stochastic matrices. We first introduce a class of $n d_{1}^{*} \times n d_{1}^{*}$ stochastic matrix sequences motivated by the stochastic approximation based consensus algorithm with delay, and then show that their ergodicity analysis is equivalent to that of a sequence of $n \times n$ degenerating stochastic matrices. Our main idea of studying the backward products is to run a noiseless switching linear dynamical system governed by these matrices. By setting different initial conditions, the sequence of state vectors, as the output of the linear system, will reflect information on the backward products. The analysis of the state vectors is simpler than directly handling the backward products. To avoid introducing too many variables, the vectors $X_{t}$ and $\mathbf{X}_{\mathbf{t}}$ appearing in Section II will be reused in different systems and this should cause no risk of confusion.

\section{A. Compatible Matrices}

Let $\left\{\mathbf{A}_{t}, t \geq 0\right\}$ be $n d_{1}^{*} \times n d_{1}^{*}$ deterministic nonnegative matrices, where $d_{1}^{*}=d^{*}+1$. Each $\mathbf{A}_{t}$ is a stochastic matrix of the form

$$
\begin{aligned}
& \mathbf{A}_{t}=\left[\begin{array}{ccc}
A_{00, t} & \ldots & A_{0 d^{*}, t} \\
\vdots & \ddots & \vdots \\
A_{d^{*} 0, t} & \ldots & A_{d^{*} d^{*}, t}
\end{array}\right] \\
& =\left[\begin{array}{c|c}
A_{00, t}, \ldots, A_{0\left(d^{*}-1\right), t} & A_{0 d^{*}, t} \\
\hline I & 0_{n d^{*} \times n}
\end{array}\right]
\end{aligned}
$$


where each matrix $A_{i k, t}$ is $n \times n$ and the identity matrix is $n d^{*} \times n d^{*}$. Let $\mathbf{A}_{t}(i, j)$ be the $(i, j)$ th element of $\mathbf{A}_{t}$. We consider a class of stochastic matrix sequences motivated by the consensus algorithm (15). When $t$ is large, each $\mathbf{A}_{\mathbf{t}}$ is nearly a $0-1$ matrix. However, these nearly zero elements still have an important effect on the asymptotic property of the backward products. Our idea is to introduce the notion of compatible matrices by associating $\mathbf{A}_{t}$ with a digraph $\tilde{G}_{t}$ where the latter identifies some relatively strong transitions described by $\mathbf{A}_{t}$. To facilitate further analysis, this compatibility notion is also defined for $n \times n$ matrices.

Definition 4: Let $\left\{\delta_{t}, t \geq 0\right\}$ be a sequence of nonnegative numbers with $\lim _{t \rightarrow \infty} \delta_{t}=0$ and $\left\{\tilde{G}_{t}=\left(\mathcal{N}, \tilde{\mathcal{E}}_{t}\right), t \geq 0\right\}$ be a sequence of digraphs of $n$ nodes.

a) The sequence of $n d_{1}^{*} \times n d_{1}^{*}$ stochastic matrices $\left\{\mathbf{A}_{t}, t \geq\right.$ $0\}$ of the form (17) is said to be $\left(\delta_{t}\right)$-compatible with $\left\{\tilde{G}_{t}, t \geq 0\right\}$ if there exist constants $t_{c}, 0<\underline{c} \leq \bar{c}$ such that for all $t \geq t_{c}$

$$
\begin{gathered}
\mathbf{A}_{t}(i, j) \leq \bar{c} \delta_{t}, \quad \forall 1 \leq i \leq n, \quad 1 \leq j \leq n d_{1}^{*}, \quad i \neq j, \\
\max _{0 \leq d \leq d^{*}} \mathbf{A}_{t}(i, j+d n) \geq \underline{c} \delta_{t}, \quad \forall(j, i) \in \tilde{\mathcal{E}}_{t} .
\end{gathered}
$$

b) The sequence of $n \times n$ stochastic matrices $\left\{M_{t}, t \geq 0\right\}$ is said to be $\left(\delta_{t}\right)$-compatible with $\left\{\tilde{G}_{t}, t \geq 0\right\}$ if there exist $t_{c}, 0<\underline{c} \leq \bar{c}$ such that

$$
\begin{array}{ll}
M_{t}(i, j) \leq \bar{c} \delta_{t}, & \forall 1 \leq i, j \leq n, \quad i \neq j, \\
M_{t}(i, j) \geq \underline{c} \delta_{t}, \quad \forall(j, i) \in \tilde{\mathcal{E}}_{t}
\end{array}
$$

where $M_{t}(i, j)$ is the $(i, j)$ th element of $M_{t}$.

Remark: We may further define $\left(\delta_{t}\right)$-compatibility with any positive initial time $t_{0}$ in an obvious manner. If $d^{*}=0$, part a) of Definition 4 reduces to part b). If $\left\{\mathbf{A}_{t}, t \geq 0\right\}$ is $\left(\delta_{t}\right)$-compatible with $\left\{\tilde{G}_{t}, t \geq 0\right\}$, this property still holds if $\delta_{t}$ is replaced by $c \delta_{t}$ for some $c>0$.

Example: Take $\mathcal{N}=\{1,2\}, \mathcal{E}_{0}=\{(2,1)\}$ and $\mathcal{E}_{1}=\{(1,2),(2,1)\}$. Let $\left\{\tilde{G}_{t}, t \geq 1\right\}$ be defined by $\tilde{G}_{2 k}=\left(\mathcal{N}, \mathcal{E}_{0}\right)$ and $\tilde{G}_{2 k-1}=\left(\mathcal{N}, \mathcal{E}_{1}\right)$ for $k \geq 1$. Let

$$
\begin{aligned}
\mathbf{A}_{2 k} & =\left[\begin{array}{cccc}
1-\frac{1}{k} & 0 & \frac{3}{4 k} & \frac{1}{4 k} \\
\frac{1}{2 k^{2}} & 1-\frac{1}{k^{2}} & \frac{1}{2 k^{2}} & 0 \\
1 & 0 & 0 & 0 \\
0 & 1 & 0 & 0
\end{array}\right], \\
\mathbf{A}_{2 k-1} & =\left[\begin{array}{cccc}
1-\frac{1}{k} & \frac{1}{2 k} & 0 & \frac{1}{2 k} \\
\frac{1}{2 k} & 1-\frac{1}{k} & \frac{1}{2 k} & 0 \\
1 & 0 & 0 & 0 \\
0 & 1 & 0 & 0
\end{array}\right] .
\end{aligned}
$$

It is easy to verify that $\left\{\mathbf{A}_{t}, t \geq 1\right\}$ is $(1 / t)$-compatible with $\left\{\tilde{G}_{t}, t \geq 1\right\}$, where we take $\bar{c}=2$ and $\underline{c}=1 / 3$ for conditions (18), (19). For instance, when $t=2 k$, node 2 is a neighbor of node 1. In relation to (19), we check the first row of $\mathbf{A}_{2 k}$, and the maximum of its second and fourth elements is $1 /(4 k) \geq$ $\underline{c} /(2 k)$.

Example: Let $\left\{G_{t}(\omega), t \geq 0\right\}$ be given in Section II and $\left\{B_{t}(\omega), t \geq 0\right\}$ be specified by (5), (6), where $\omega \in \Omega$ is fixed.
Then $\left\{\mathbf{A}_{t}(\omega):=\mathbf{U}+a_{t} \mathbf{B}_{t}(\omega), t \geq t_{0}(\omega)\right\}$ is $\left(a_{t}\right)$-compatible with $\left\{G_{t}(\omega), t \geq t_{0}(\omega)\right\}$ if $\left\{a_{t}, t \geq 0\right\}$ satisfies (A1) and $\mathbf{A}_{t}(\omega)$ is a nonnegative matrix for $t \geq t_{0}(\omega)$.

Given $\mathbf{A}_{t}$ in (17), define

$$
M_{t}^{A}=\sum_{d=0}^{d^{*}} A_{0 d, t}
$$

which is a stochastic matrix. The following property is obvious and the proof is omitted.

Proposition 5: Let $\left\{M_{t}^{A}, t \geq 0\right\}$ be defined by (22). If $\left\{\mathbf{A}_{t}, t \geq 0\right\}$ is $\left(\delta_{t}\right)$-compatible with $\left\{\tilde{G}_{t}, t \geq 0\right\}$, then so is $\left\{M_{t}^{A}, t \geq 0\right\}$.

The backward products of $\left\{\mathbf{A}_{t}, t \geq 0\right\}$ are closely related to the weighted averaging algorithm

$$
\mathbf{X}_{t+1}=\mathbf{A}_{t} \mathbf{X}_{t}, \quad t \geq 0 .
$$

To distinguish from the notation in (15), for (23) we denote

$$
\mathbf{X}_{t}=\left[X_{t} ; X_{t}^{(-1)} ; \ldots ; X_{t}^{\left(-d^{*}\right)}\right]
$$

where $X_{t}$ and $X_{t}^{(-d)}, 1 \leq d \leq d^{*}$, are $n$ dimensional. Further denote the $n$-dimensional linear system

$$
X_{t+1}=M_{t}^{A} X_{t}, \quad t \geq 0 .
$$

With the aid of the linear systems (23) and (25), we may prove the following equivalence theorem.

Theorem 6: Assume that $\left\{\mathbf{A}_{t}, t \geq 0\right\}$ is $\left(\delta_{t}\right)$-compatible with $\left\{\tilde{G}_{t}, t \geq 0\right\}$ and $\sum_{t=0}^{\infty} \delta_{t}^{2}<\infty$. Let $\left\{M_{t}^{A}, t \geq 0\right\}$ be defined by (22). Then $\left\{\mathbf{A}_{t}, t \geq 0\right\}$ has ergodic backward products if and only if $\left\{M_{t}^{A}, t \geq 0\right\}$ has ergodic backward products.

Proof: See Appendix B.

The important implication of Theorem 6 is that the ergodicity property of the delay based sequence $\left\{\mathbf{A}_{t}, t \geq 0\right\}$ may be studied via a lower dimensional sequence without involving delay.

\section{B. Linear Systems Governed by $n \times n$ Compatible Nonnegative Matrices}

With Theorem 6 in mind, below we focus on analyzing $n \times n$ stochastic matrices. For a sequence of $n \times n$ stochastic matrices $\left\{M_{t}, t \geq 0\right\}$, consider the linear system

$$
X_{t+1}=M_{t} X_{t}, \quad t \geq 0
$$

which may be interpreted as a consensus algorithm over a digraph $G_{M, t}$ of $n$ nodes. The edges of $G_{M, t}$ are uniquely determined by the nonzero off-diagonal elements in $M_{t}$. We introduce the assumption.

(H1) i) $\left\{M_{t}, t \geq 0\right\}$ is $\left(a_{t}\right)$-compatible with a sequence of digraphs $\left\{\tilde{G}_{t}, t \geq 0\right\}$, where $\left\{a_{t}, t \geq 0\right\}$ satisfies (A1); ii) each $\tilde{G}_{t}$ is strongly connected.

Remark: $\tilde{G}_{t}$ may be different from $G_{M, t}$.

\section{State Reordering and Mutual Attraction of Trajectories}

We run (26) with any fixed initial pair $\left(t_{0}, X_{t_{0}}\right)$, where $t_{0} \geq 0$. If $X_{t}$ is considered directly for convergence analysis, 
the problem is quite difficult since the components of $X_{t}$ undergo very complex evolution. We define a new vector $Z_{t}$ by reordering the $n$ entries in $X_{t}=\left[x_{t}^{1}, \ldots, x_{t}^{n}\right]^{T}$. Suppose

$$
x_{t}^{i_{1}} \geq x_{t}^{i_{2}} \geq \ldots \geq x_{t}^{i_{n}}
$$

where $\left\{i_{1}, \ldots, i_{n}\right\}$ is a permutation of $\{1, \ldots, n\}$ and in general changes with time. We interpret $x_{t}^{i}$ as the state of node $i, 1 \leq$ $i \leq n$. Define

$$
Z_{t}=\left[z_{t}^{1}, \ldots, z_{t}^{n}\right]^{T}:=\left[x_{t}^{i_{1}}, \ldots, x_{t}^{i_{n}}\right]^{T} .
$$

After reordering of the states, the evolution of $Z_{t}$ has more orderly behavior than that of $X_{t}$.

In later analysis, we need to identify a component of $Z_{t}$ as a component of $X_{t}$ and further check its recursive equation. We use the following rule to avoid indeterminacy when the same value repeats within $Z_{t}$. If there are exactly $l \geq 2$ components $z_{t}^{k}, \ldots, z_{t}^{k+l-1}$ within $Z_{t}$ taking the same value $c_{l}$ corresponding to $l$ nodes with indices $i_{1}<i_{2}<\ldots<i_{l}$, the $k$ th component $z_{t}^{k}$ in $Z_{t}$ is interpreted as the state of the node with the smallest index $i_{1}$; similarly, the $(k+1)$ th component in $Z_{t}$ is associated with the second smallest node index $i_{2}$, and so on. Define the $n$ scalar sequences

$$
\left\{z_{t}^{k}, t \geq t_{0}\right\}, \quad 1 \leq k \leq n .
$$

We call $\left\{z_{t}^{k}, t \geq t_{0}\right\}$ the level- $k$ trajectory. In analyzing $\left\{Z_{t}, t \geq t_{0}\right\}$, the basic idea is to show that there is a mutual attraction of the trajectories of different levels, which eventually merge to the same limit. Due to the degenerating stochastic matrices, our method differs from directly comparing the gap between the greatest and least states in a consensus algorithm with time varying weight matrices [6].

Since each $M_{t}$ is a stochastic matrix, $\left\{z_{t}^{1}, t \geq t_{0}\right\}$ (resp., $\left\{z_{t}^{n}, t \geq t_{0}\right\}$ ) is bounded and non-increasing (resp., non-decreasing) (see, e.g., [6]), and so has a limit. We summarize this fact in the following proposition.

Proposition 7: Let $Z_{t}$ be defined by (26), (27). Then both $\left\{z_{t}^{1}, t \geq t_{0}\right\}$ and $\left\{z_{t}^{n}, t \geq t_{0}\right\}$ converge to finite limits.

The asymptotic behavior of the other sequences $\left\{z_{t}^{k}, t \geq t_{0}\right\}$, where $k \in\{2, \ldots, n-1\}$, is less obvious. The following theorem proves one of the key results of this paper. It is instrumental for establishing ergodicity of backward products of degenerating stochastic matrices. Its proof is quite involved. The basic idea is to use induction. First, the level-1 trajectory converges to a finite limit. Next, we show that each level- $(k+1)$ trajectory converges to the same limit as the level- $k$ trajectory.

Theorem 8: Let $\left(X_{t}, Z_{t}\right)$ be defined by (26), (27) with any initial condition $X_{t_{0}}, t_{0} \geq 0$, and assume (H1). Then there exists $c \in \mathbb{R}$ such that $\lim _{t \rightarrow \infty} Z_{t}=\lim _{t \rightarrow \infty} X_{t}=c \mathbf{1}_{n}$.

Proof: See Appendix C.

Remark: The proof of Theorem 8 only requires $\gamma \in(0,1]$ for (10). The further restriction $\gamma \in(1 / 2,1]$ is needed for proving mean square convergence in Section VI.

\section{Leader Following}

A leader following structure may be incorporated into (26), and in this case (H1) is replaced by the following assumption.
(H1' $)$ i) $\left\{M_{t}, t \geq 0\right\}$ is $\left(a_{t}\right)$-compatible with a sequence of digraphs $\left\{\tilde{G}_{t}, t \geq 0\right\}$, where $\left\{a_{t}, t \geq 0\right\}$ satisfies (A1); ii) $M\left(i_{L}, i_{L}\right)=1$; iii) each $\tilde{G}_{t}$ contains a spanning tree, and all these spanning trees share a common root $i_{L}$ which has no neighbor in $\tilde{G}_{t}$.

(H1')-ii) ensures that the $i_{L}$ th element of $X_{t}$ is fixed as the leader' state $x_{0}^{i_{L}}$.

Our strategy of proving convergence for the leader following model is to start with a special class of initial conditions of $X_{t}$ so that we may adapt the argument in proving Theorem 8 which has been based on jointly strongly connected digraphs. To treat general initial conditions, we apply a transformation of the initial condition so that the analysis is reduced to the previous case.

Corollary 9: Let $X_{t}$ be defined by (26) with any initial condition $X_{t_{0}}, t_{0} \geq 0$. Assuming $\left(\mathbf{H 1}^{\prime}\right)$, then $\lim _{t \rightarrow \infty} X_{t}=x_{t_{0}}^{i_{L}} \mathbf{1}_{n}$.

Proof: By (H1')-ii), it follows that $x_{t}^{i_{L}}=x_{t_{0}}^{i_{L}}$ for $t \geq t_{0}$. Take a sufficiently large $t_{1} \geq t_{0}+1$ such that by (20) after $\delta_{t}$ is replaced by $a_{t}$

$$
\min _{1 \leq i \leq n} M_{t}(i, i)>\frac{1}{2}, \quad \forall t \geq t_{1} .
$$

Now let $X_{t_{1}}$ be generated with the initial pair $\left(t_{0}, X_{t_{0}}\right)$ and used as the initial condition for

$$
X_{t+1}=M_{t} X_{t}, \quad t \geq t_{1} .
$$

Proposition 7 still holds in the leader following case.

Step 1) Assume that for (30)

$$
x_{t_{0}}^{i_{L}}=x_{t_{1}}^{i_{L}}<x_{t_{1}}^{j}, \quad \forall j \in S_{F}=\{1, \ldots, n\} \backslash\left\{i_{L}\right\} .
$$

By (29) and induction it is straightforward to show that $x_{t}^{j}>x_{t_{0}}^{i_{L}}$ for $j \in S_{F}$ and all $t \geq t_{1}$. Hence, $x_{t}^{i_{L}}<x_{t}^{j}$ for $j \in S_{F}$ and $t \geq t_{1}$. Following (27), we still reorder the $n$ components of $X_{t}$ in (30) from the greatest to the least to obtain the vector $Z_{t}, t \geq t_{1}$. So $z_{t}^{n}=x_{t}^{i_{L}}$ for $t \geq t_{1}$.

First, by Proposition $7,\left\{z_{t}^{1}, t \geq t_{1}\right\}$ has a finite limit $c$. Next, by using the same induction argument as in proving Theorem 8 , we may show that all the $n$ sequences $\left\{z_{t}^{k}, t \geq t_{1}\right\}, k=1, \ldots, n$, must converge to the same limit $c$. In particular, when we apply induction, the argument for deriving (C.15) from (C.14) is still valid under $\left(\mathbf{H 1}^{\prime}\right)$-iii) since there exists at least one edge pointing to the set of nodes associated with the first $l \leq n-1$ levels of trajectories from the remaining nodes which contain the leader since $z_{t}^{n}=x_{t}^{i_{L}}$. On the other hand, since $z_{t}^{n} \equiv x_{t_{0}}^{i_{L}}$, the limit must be $c=x_{t_{0}}^{i_{L}}$. We conclude that $\lim _{t \rightarrow \infty} Z_{t}=x_{t_{0}}^{i_{L}} \mathbf{1}_{n}$, and subsequently $\lim _{t \rightarrow \infty} X_{t}=x_{t_{0}}^{i_{L}} \mathbf{1}_{n}$.

Step 2) Consider $X_{t_{1}}$ not satisfying the inequality in (31). Let $e_{i} \in \mathbb{R}^{n}$ be a unit vector with the $i$ th element equal to 1 . Select $C_{L}>0$ such that the element at the $i_{L}$ th position of $Y_{t_{1}}=X_{t_{1}}-C_{L} e_{i_{L}}$ is less than any other element. Take $X_{t_{1}}^{\prime}=Y_{t_{1}}$ as the initial condition of

$$
X_{t+1}^{\prime}=M_{t} X_{t}^{\prime}, \quad t \geq t_{1} .
$$


By Step $1, \lim _{t \rightarrow \infty} X_{t}^{\prime}=\left(x_{t_{0}}^{i_{L}}-C_{L}\right) \mathbf{1}_{n}$. Take the initial condition $X_{t_{1}}^{\prime \prime}=-C_{L} e_{i_{L}}$ for

$$
X_{t+1}^{\prime \prime}=M_{t} X_{t}^{\prime \prime}, \quad t \geq t_{1} .
$$

By Step $1, \lim _{t \rightarrow \infty} X_{t}^{\prime \prime}=-C_{L} \mathbf{1}_{n}$. By linearity of (32), (33), $X_{t}=X_{t}^{\prime}-X_{t}^{\prime \prime}, t \geq t_{1}$, where $X_{t}$ is generated by (30). Hence, $\lim _{t \rightarrow \infty} X_{t}=x_{t_{0}}^{i_{L}} \mathbf{1}_{n}$.

\section{ERgodicity of Degenerating Stochastic Matrices}

Our plan of proving ergodicity is as follows. For the simple case where $\left\{M_{t}, t \geq 0\right\}$ in (26) is compatible with a sequence of strongly connected digraphs $\left\{\tilde{G}_{t}, t \geq 0\right\}$, the convergence result in Theorem 8 is applicable. For the general case with joint connectivity, our strategy is to reduce to the simple case. We select a sequence of times $0=: \tau_{0}<\tau_{1}<\ldots$ to form a union of digraphs and a backward sub-product on each subinterval $\left[\tau_{i}, \tau_{i+1}\right)$, where each union of digraphs is strongly connected. This is a typical procedure to exploit joint connectivity; see, e.g., [20] and [36]. Then the original backward product may be written as the product of these sub-products, possibly together with extra multiplicative terms at two ends. A key step is to show that the sub-products have desired properties to generate compatible stochastic matrices (see Lemmas D.1 and D.2).

Throughout Sections V-A and V-B, all matrices and digraphs are deterministic.

\section{A. Ergodicity of Backward Products}

For the sequence $\left\{M_{t}, t \geq 0\right\}$, define the backward product

$$
\Phi_{t, s}=M_{t-1} \ldots M_{s}, \quad t>s \geq 0
$$

and $\Phi_{s, s}:=I$. The following ergodicity theorem is an easy consequence of Theorem 8 .

Theorem 10: Assuming (H1), ergodicity holds for the backward products of $\left\{M_{t}, t \geq 0\right\}$.

Proof: Let $e_{i} \in \mathbb{R}^{n}$ be the unit column vector with the $i$ th element equal to 1 . For any fixed $s \geq 0$, by Theorem 8 there exists $\pi_{i}(s)$ depending on $s$ such that $\lim _{t \rightarrow \infty} \Phi_{t, s} e_{i}=$ $\pi_{i}(s) \mathbf{1}_{n}$. By Lemma B.1, the theorem follows.

To generalize Theorem 10, we consider ergodicity for stochastic matrices associated with jointly strongly connected digraphs. For a sequence of digraphs $\left\{\tilde{G}_{t}=\left(\mathcal{N}, \tilde{\mathcal{E}}_{t}\right), t \geq 0\right\}$, we follow the rule in Section II to define the union of digraphs. For two integers $0 \leq \tau_{t}<\tau_{t+1}$, define

$$
\widehat{M}_{t}=M_{\tau_{t+1}-1} \ldots M_{\tau_{t}}, \quad \widehat{G}_{t}=\tilde{G}_{\left[\tau_{t}, \tau_{t+1}\right)} .
$$

Theorem 11: Assume i) $\left\{M_{t}, t \geq 0\right\}$ is $\left(a_{t}\right)$-compatible with $\left\{\tilde{G}_{t}, t \geq 0\right\}$, where $\left\{a_{t}, t \geq 0\right\}$ satisfies (A1); ii) the sequence $0=: \tau_{0}<\tau_{1}<\ldots$ satisfies $\sup _{i \geq 0}\left(\tau_{i+1}-\tau_{i}\right)<\infty$; and iii) $\tilde{G}_{\left[\tau_{i}, \tau_{i+1}\right)}$ is strongly connected for each $i \geq 0$. Then ergodicity holds for the backward products of $\left\{M_{t}, t \geq 0\right\}$.

Proof: By Lemma D.2 and Theorem 10, the backward products of $\left\{\widehat{M}_{t}, t \geq 0\right\}$ are ergodic. For any $s \geq 0$, there exists some $i_{0} \geq 1$ such that $\tau_{i_{0}-1} \leq s<\tau_{i_{0}}$. Denote $\Pi_{0}=$ $\lim _{t \rightarrow \infty} \widehat{M}_{t} \ldots \widehat{M}_{i_{0}}$, which is a stochastic matrix of identical rows. For any $\epsilon>0$, there exists $K_{0}>i_{0}$ such that

$$
\left|\widehat{M}_{l} \ldots \widehat{M}_{i_{0}}-\Pi_{0}\right| \leq \epsilon
$$

for all $l \geq K_{0}$. Now for all $t \geq \tau_{K_{0}+1}+1$, we have

$$
\begin{aligned}
M_{t-1} & \ldots M_{s} \\
= & M_{t-1} \ldots M_{\tau_{K_{0}+1}} \widehat{M}_{K_{0}} \ldots \widehat{M}_{i_{0}} M_{\tau_{i_{0}}-1} \ldots M_{s} \\
= & M_{t-1} \ldots M_{\tau_{K_{0}+1}}\left[\widehat{M}_{K_{0}} \ldots \widehat{M}_{i_{0}}-\Pi_{0}\right] M_{\tau_{i_{0}}-1} \ldots M_{s} \\
& +M_{t-1} \ldots M_{\tau_{K_{0}+1}} \Pi_{0} M_{\tau_{i_{0}}-1} \ldots M_{s} \\
= & M_{t-1} \ldots M_{\tau_{K_{0}+1}}\left[\widehat{M}_{K_{0}} \ldots \widehat{M}_{i_{0}}-\Pi_{0}\right] M_{\tau_{i_{0}}-1} \ldots M_{s} \\
& +\Pi_{0} M_{\tau_{i_{0}}-1} \ldots M_{s}
\end{aligned}
$$

where $\Pi_{0} M_{\tau_{i_{0}}-1} \ldots M_{s}$ is a stochastic matrix of identical rows. Since $M_{t-1} \ldots M_{\tau_{K_{0}+1}}$ and $M_{\tau_{i_{0}}-1} \ldots M_{s}$ are stochastic matrices, $\mid M_{t-1} \ldots M_{\tau_{K_{0}+1}}\left[\widehat{M}_{K_{0}} \ldots \widehat{M}_{i_{0}}-\right.$ $\left.\Pi_{0}\right] M_{\tau_{i_{0}}-1} \ldots M_{s} \mid \leq n \epsilon$. Since $\epsilon>0$ is arbitrary, $\lim _{t \rightarrow \infty} \Phi_{t, s}=\Pi_{0} M_{\tau_{i_{0}}-1} \ldots M_{s}$.

Remark: Theorems 10 and 11 hold with $\gamma \in(0,1]$ for $(10)$ since the proofs of Theorem 8 and Lemma D.1 only need $\gamma \in$ $(0,1]$.

\section{B. Backward Products of $\left\{\mathbf{A}_{t}, t \geq 0\right\}$}

Theorem 12: Assume i) $\left\{\mathbf{A}_{t}, t \geq 0\right\}$ is $\left(a_{t}\right)$-compatible with $\left\{\tilde{G}_{t}, t \geq 0\right\}$, where $\left\{a_{t}, t \geq 0\right\}$ satisfies (A1); ii) the sequence $0=: \tau_{0}<\tau_{1}<\ldots$ satisfies $\sup _{i \geq 0}\left(\tau_{i+1}-\tau_{i}\right)<\infty$; and iii) $\tilde{G}_{\left[\tau_{i}, \tau_{i+1}\right)}$ is strongly connected for each $i \geq 0$. Then ergodicity holds for the backward products of $\left\{\mathbf{A}_{t}, t \geq 0\right\}$.

Proof: By Proposition 5, $\left\{M_{t}^{A}, t \geq 0\right\}$ is $\left(a_{t}\right)$-compatible with $\left\{\tilde{G}_{t}, t \geq 0\right\}$, and so has ergodic backward products by Theorem 11. By Theorem 6, $\left\{\mathbf{A}_{t}, t \geq 0\right\}$ has ergodic backward products.

\section{Application to Random Networks}

In (A2), we may select a null set $N_{0}$ (i.e., $P\left(N_{0}\right)=0$ ) such that $G_{\left[T_{l}(\omega), T_{l+1}(\omega)\right)}(\omega)$ is strongly connected and $\alpha_{1}(\omega)=$ $\sup _{l \geq 0}\left|T_{l+1}(\omega)-T_{l}(\omega)\right|<\infty$ for $\omega \in \Omega \backslash N_{0}$.

Corollary 13: Assume i) $\left\{B_{t}, t \geq 1\right\}$ is given by (5), (6) and (A1)-(A2) hold; ii) $s_{0}(\omega)$ is an integer such that each $\mathbf{A}_{t}^{\dagger}=$ $\mathbf{U}+\mathbf{a}_{\theta_{t}} \mathbf{B}_{t}, t \geq s_{0}(\omega)$, is a stochastic matrix. Then for each $\omega \in \Omega \backslash N_{0}$, ergodicity holds for the backward products of $\left\{\mathbf{A}_{t}^{\dagger}(\omega), t \geq s_{0}(\omega)\right\}$.

Proof: For synchronous step size update, $\left\{\mathbf{A}_{t}^{\dagger}(\omega), t \geq\right.$ $\left.s_{0}(\omega)\right\}$ is $\left(a_{t}\right)$-compatible with $\left\{G_{t}(\omega), t \geq s_{0}(\omega)\right\}$.

We further check the compatibility condition with asynchronous step size update. Take $\omega \in \Omega \backslash N_{0}$. We have $1 \leq \alpha_{1}(\omega)<\infty$. Consider any node $i_{0}$ and integer $l \geq 1$. Since $G_{\left[T_{l}(\omega), T_{l+1}(\omega)\right)}(\omega)$ is strongly connected, there exists at least one node $j_{0}$ as a neighbor of node $i_{0}$ in $G_{\left[T_{l}(\omega), T_{l+1}(\omega)\right)}(\omega)$. Suppose that $\left(j_{0}, i_{0}\right)$ is an edge in $G_{T_{l}(\omega)+\xi}(\omega)$, where $0 \leq \xi<T_{l+1}(\omega)-T_{l}(\omega)$. Then

$$
\theta_{T_{l}(\omega)-1}^{i_{0}}(\omega)+1 \leq \theta_{T_{l+1}(\omega)-1}^{i_{0}}(\omega) \leq \theta_{T_{l}(\omega)-1}^{i_{0}}(\omega)+\alpha_{1}(\omega)
$$

where the second inequality follows from $\theta_{k+j}^{i_{0}} \leq \theta_{k}^{i_{0}}+j$ for $j \geq 1$. Since $\left\{\theta_{t}^{i_{0}}, t \geq 0\right\}$ is non-decreasing, by (35) there exist coefficients $0<c_{1}(\omega) \leq c_{2}(\omega)$ such that

$$
c_{1}(\omega) t \leq \theta_{t}^{i_{0}}(\omega) \leq c_{2}(\omega) t
$$


for all $t \geq T_{1}(\omega)$. Subsequently,

$$
\alpha\left[c_{2}(\omega) t\right]^{-\gamma} \leq a_{\theta_{t}^{i_{0}}(\omega)} \leq \beta\left[c_{1}(\omega) t\right]^{-\gamma}, \quad t \geq T_{1}(\omega)
$$

Next, for $\omega \in \Omega \backslash N_{0}$, by (36) we may check that $\left\{\mathbf{A}_{t}^{\dagger}(\omega), t \geq\right.$ $\left.s_{0}(\omega)\right\}$ is $\left(a_{t}\right)$-compatible with $\left\{G_{t}(\omega), t \geq s_{0}(\omega)\right\}$. The corollary follows from Theorem 12 .

\section{Mean Souare Consensus}

Let (15) be rewritten in the form

$$
\mathbf{X}_{t+1}=\mathbf{A}_{t}^{\dagger} \mathbf{X}_{t}+\mathbf{a}_{\theta_{t}} \mathbf{D}_{t} W_{t}, \quad t \geq 0
$$

where $\mathbf{A}_{t}^{\dagger}=\mathbf{U}+\mathbf{a}_{\theta_{t}} \mathbf{B}_{t}$. By (9), (12) and (14), $\mathbf{D}_{t}$ is a function of $B_{t}$, and $\sup _{t \geq 0, \omega \in \Omega}\left|\mathbf{D}_{t}\right|<\infty$.

Theorem 14: Assuming (A1)-(A3), mean square consensus holds for (9) with synchronous step size update, i.e., $\lim _{t \rightarrow \infty} E\left|x_{t}^{i}-x^{*}\right|^{2}=0$ for some $x^{*}$ and for all $1 \leq i \leq n$.

Proof: We have $\mathbf{a}_{\theta_{t}}=\operatorname{Diag}\left(a_{t} I_{n}, 0_{n d^{*} \times n d^{*}}\right)$ and $\left\{W_{t}, t \geq 0\right\}$ is independent of $\left\{\left(\mathbf{A}_{t}^{\dagger}, \mathbf{D}_{t}\right), t \geq 0\right\}$. Take a sufficiently large $t_{0}$ such that w.p. $1 \mathbf{A}_{t}^{\dagger}$ is a stochastic matrix for $t \geq t_{0}$. In addition,

$$
\sum_{t=0}^{\infty} E\left|\mathbf{a}_{\theta_{t}} \mathbf{D}_{t}\right|^{2} E\left|W_{t}\right|^{2}=\sum_{t=0}^{\infty} a_{t}^{2} E\left|D_{t}\right|^{2} E\left|W_{t}\right|^{2}<\infty
$$

The theorem follows from Theorem 3 and Corollary 13 .

Theorem 15: Assume (A1)-(A3). In addition, i) there exists a deterministic integer $t_{0}$ such that w.p. $1 \mathbf{A}_{t}^{\dagger}$ is a nonnegative matrix for all $t \geq t_{0}$; ii) $E \sup _{l>1} \Delta_{l}^{2 \gamma}<\infty$, where $\Delta_{l}=T_{l+1}-T_{l}$. Then algorithm (9) with asynchronous step size update ensures mean square consensus.

Proof: For the asynchronous case, $\mathbf{a}_{\theta_{t}}$ is a matrix function of the adjacency matrices $\left(A_{G_{k}}, 0 \leq k \leq t\right)$. It is easy to see that $\left\{W_{t}, t \geq 0\right\}$ is independent of $\left\{\left(\mathbf{A}_{t}^{\dagger}, \mathbf{a}_{\theta_{t}} \mathbf{D}_{t}\right), t \geq 0\right\}$. For some constant $C$, we have

$\sum_{t=0}^{\infty} E\left|\mathbf{a}_{\theta_{t}} \mathbf{D}_{t}\right|^{2} E\left|W_{t}\right|^{2} \leq C \sum_{t=0}^{\infty} E\left|\mathbf{a}_{\theta_{t}}\right|^{2}=C \sum_{t=0}^{\infty} \sum_{i=1}^{n} E\left|a_{\theta_{t}^{i}}\right|^{2}$.

For some constant $C_{0}, a_{\theta_{t}^{i}} \leq C_{0} \sup _{l \geq 1} \Delta_{l}^{\gamma} / t^{\gamma}$ for $t \geq 1$ since $\theta_{t}^{i} \geq\left(t / \sup _{l \geq 1} \Delta_{l}\right)-1$. Subsequently,

$\sum_{t=0}^{\infty} E\left|\mathbf{a}_{\theta_{t}} \mathbf{D}_{t}\right|^{2} E\left|W_{t}\right|^{2} \leq C_{1} a_{0}^{2}+C_{1} E \sup _{l \geq 1} \Delta_{l}^{2 \gamma} \sum_{t=1}^{\infty} t^{-2 \gamma}<\infty$.

The theorem follows from Theorem 3 and Corollary 13 .

Remark: If $\sup _{l>1} \Delta_{l} \leq C$ w.p.1 for some constant $C$, conditions i) and ii) in Theorem 15 hold.

For leader following, if node $i_{L}$ is the leader, the $i_{L}$ th row of $D_{t}$ has all zeros for each $t$ due to (9). The convergence for the leader following case may be proved by an ergodicity approach based on Corollary 13. The proof is omitted.
Corollary 16: In Theorems 14 and 15, if (A2) is replaced by $\left(\mathbf{A} \mathbf{2}^{\prime}\right)$ while other assumptions still hold, then $\lim _{t \rightarrow \infty} E\left|x_{t}^{i}-x_{0}^{i_{L}}\right|^{2}=0$ for all $i$.

Remark: Theorems 14-15 and Corollary 16 may be generalized to correlated noises by letting $\left\{W_{t}, t \geq 0\right\}$ satisfy condition iii) in Theorem 3.

Remark: If (3) is replaced by $y_{t}^{i k}=\left.\left(x_{s}^{k}+w_{s}^{i k}\right)\right|_{s=t-d_{t}^{i k}}$, Theorems 14-15 and Corollary 16 still hold.

\section{CONCLUDING REMARKS}

We considered synchronous and asynchronous stochastic approximation for consensus seeking with delayed measurements in dynamic noisy environments. This paper developed ergodicity results for degenerating stochastic matrices and proved mean square consensus without quadratic Lyapunov functions. In future work, it will be of interest to relax the bounded time interval condition for joint connectivity so that the modeling may deal with more general random networks, such as Markovian switching networks [29], [17]. Convergence rate bounds and probability one convergence of the consensus algorithm are also interesting topics.

\section{APPENDIX A}

PROOF OF THEOREM 3

Lemma A.1: Denote $\xi_{T, j}=\sum_{k=T}^{T+j-1} \Psi_{T+j, k+1} H_{k} V_{k}$ for $T \geq 0$ and $j \geq 1$. Then

$$
E\left|\xi_{T, j}\right|^{2} \leq 2 m_{1} m_{2} \sum_{l=0}^{\infty} \phi_{l} \sum_{k=T}^{T+j-1} E\left|H_{k}\right|^{2} E\left|V_{k}\right|^{2}
$$

Proof: For all $\omega$ and $t \geq s, \Psi:=\Psi_{t, s}(\omega)$ is a stochastic matrix ensuring $|\Psi|^{2}=\operatorname{Tr}\left(\Psi^{T} \Psi\right)=\operatorname{Tr}\left(\Psi \Psi^{T}\right) \leq m_{1}$. We have

$$
E\left|\xi_{T, j}\right|^{2}=\sum_{T \leq i, k \leq T+j-1} E\left[V_{i}^{T} H_{i}^{T} \Psi_{T+j, i+1}^{T} \Psi_{T+j, k+1} H_{k} V_{k}\right]
$$

For each pair $(i, k)$

$$
\begin{aligned}
& \left|E\left[V_{i}^{T} H_{i}^{T} \Psi_{T+j, i+1}^{T} \Psi_{T+j, k+1} H_{k} V_{k}\right]\right| \\
& \quad=\left|\operatorname{Tr}\left\{E\left[H_{i}^{T} \Psi_{T+j, i+1}^{T} \Psi_{T+j, k+1} H_{k}\right] E\left[V_{k} V_{i}^{T}\right]\right\}\right| \\
& \quad \leq m_{2}\left|E\left[H_{i}^{T} \Psi_{T+j, i+1}^{T} \Psi_{T+j, k+1} H_{k}\right] E\left[V_{k} V_{i}^{T}\right]\right| \\
& \quad \leq m_{2} E\left|H_{i}^{T} \Psi_{T+j, i+1}^{T} \Psi_{T+j, k+1} H_{k}\right| \cdot\left|E\left[V_{k} V_{i}^{T}\right]\right| \\
& \quad \leq m_{1} m_{2} \phi_{|k-i|} E\left(\left|H_{i}^{T}\right| \cdot\left|H_{k}\right|\right)\left(E\left|V_{k}\right|^{2} E\left|V_{i}\right|^{2}\right)^{\frac{1}{2}} \\
& \quad \leq m_{1} m_{2} \phi_{|k-i|}\left(E\left|H_{i}\right|^{2} E\left|H_{k}\right|^{2}\right)^{\frac{1}{2}}\left(E\left|V_{k}\right|^{2} E\left|V_{i}\right|^{2}\right)^{\frac{1}{2}} \\
& \quad \leq\left(\frac{m_{1} m_{2}}{2}\right) \phi_{|k-i|}\left(E\left|H_{i}\right|^{2} E\left|V_{i}\right|^{2}+E\left|H_{k}\right|^{2} E\left|V_{k}\right|^{2}\right) .
\end{aligned}
$$

Hence, by (A.1)

$$
\left|\sum_{T \leq i, k \leq T+j-1} E\left[V_{i}^{T} H_{i}^{T} \Psi_{T+j, i+1}^{T} \Psi_{T+j, k+1} H_{k} V_{k}\right]\right|
$$




$$
\begin{aligned}
& \leq\left(\frac{m_{1} m_{2}}{2}\right) \sum_{l=-j+1}^{j-1} \phi_{|l|} \sum_{k=T}^{T+j-1} 2 E\left|H_{k}\right|^{2} E\left|V_{k}\right|^{2} \\
& \leq 2 m_{1} m_{2} \sum_{l=0}^{\infty} \phi_{l} \sum_{k=T}^{T+j-1} E\left|H_{k}\right|^{2} E\left|V_{k}\right|^{2} .
\end{aligned}
$$

The lemma follows.

Proof of Theorem 3: Sufficiency-For fixed $\left(t_{0}, Y_{t_{0}}\right)$

$$
Y_{t+1}=\Psi_{t+1, t_{0}} Y_{t_{0}}+\sum_{k=t_{0}}^{t} \Psi_{t+1, k+1} H_{k} V_{k}, \quad t \geq t_{0} .
$$

By Lemma A.1, we may show that $\sup _{t \geq t_{0}} E\left|Y_{t}\right|^{2}<\infty$.

Let $\xi_{T, j}=\sum_{k=T}^{T+j-1} \Psi_{T+j, k+1} H_{k} V_{k}$ for $T \geq t_{0}$ and $j \geq 1$. For any given $\epsilon>0$, by Lemma A.1 we may select $t_{1} \geq t_{0}$ such that

$$
\sup _{T \geq t_{1}} \sup _{j \geq 1} E\left|\xi_{T, j}\right|^{2} \leq \frac{\epsilon}{2}
$$

Taking a fixed $T^{\prime} \geq t_{1}$, we have

$$
Y_{t+1}=\Psi_{t+1, T^{\prime}} Y_{T^{\prime}}+\sum_{k=T^{\prime}}^{t} \Psi_{t+1, k+1} H_{k} V_{k}, \quad t \geq T^{\prime} .
$$

Since $\Psi_{t+1, T^{\prime}}$ converges w.p. 1 to a stochastic matrix $\Pi_{T^{\prime}}$ of identical rows, there exists a random variable $\zeta_{T^{\prime}}$ such that

$$
\lim _{t \rightarrow \infty} E\left|\Psi_{t+1, T^{\prime}} Y_{T^{\prime}}-\zeta_{T^{\prime}} \mathbf{1}_{m_{1}}\right|^{2}=0 .
$$

So by (A.2), (A.3)

$$
\limsup _{t \rightarrow \infty} E\left|Y_{t+1}^{i}-Y_{t+1}^{j}\right|^{2} \leq 2 \limsup _{k \rightarrow \infty} E\left|\xi_{T^{\prime}, k}\right|^{2} \leq \epsilon
$$

and therefore, there exists $t_{2} \geq t_{1}$ such that for all $i, j$ and $t \geq t_{2}$

$$
E\left|Y_{t}^{i}-Y_{t}^{j}\right|^{2} \leq 2 \epsilon
$$

Following the argument in [7, Th. 9], we proceed to show mean square consensus. For any $t^{\prime}>t \geq t_{2}$, we have $Y_{t^{\prime}}=\Psi_{t^{\prime}, t} Y_{t}+$ $\xi_{t, t^{\prime}-t}$ and

$$
\begin{aligned}
E\left|Y_{t^{\prime}}-Y_{t}\right|^{2} \\
\quad \leq 2 E\left|\Psi_{t^{\prime}, t} Y_{t}-Y_{t}\right|^{2}+2 E\left|\xi_{t, t^{\prime}-t}\right|^{2} \\
\quad \leq 2 E\left|\left(\Psi_{t^{\prime}, t} Y_{t}^{1} \mathbf{1}_{m_{1}}-Y_{t}\right)+\Psi_{t^{\prime}, t}\left(Y_{t}-Y_{t}^{1} \mathbf{1}_{m_{1}}\right)\right|^{2}+\epsilon \\
\quad \leq 4 E\left|Y_{t}^{1} \mathbf{1}_{m_{1}}-Y_{t}\right|^{2}+4 E\left(\left|\Psi_{t^{\prime}, t}\right|^{2}\left|Y_{t}-Y_{t}^{1} \mathbf{1}_{m_{1}}\right|^{2}\right)+\epsilon \\
\quad \leq 4 m_{1}(2 \epsilon)+4 m_{1}\left(2 m_{1} \epsilon\right)+\epsilon=\left(8 m_{1}^{2}+8 m_{1}+1\right) \epsilon .
\end{aligned}
$$

Since $\epsilon>0$ is arbitrary, $\left\{Y_{t}, t \geq t_{0}\right\}$ is a Cauchy sequence in the $L_{2}$ norm and has a limit $Y_{\infty}=\left[Y_{\infty}^{1}, \ldots, Y_{\infty}^{m_{1}}\right]^{T}$. Furthermore, $E\left|Y_{\infty}^{i}-Y_{\infty}^{j}\right|^{2}=\lim _{t \rightarrow \infty} E\left|Y_{t}^{i}-Y_{t}^{j}\right|^{2}=0$ by arbitrariness of $\epsilon>0$ in (A.4). So $Y_{\infty}=Y_{\infty}^{1} \mathbf{1}_{m_{1}}$ and mean square consensus follows.

Necessity-Given any $\epsilon>0$ and $t_{0} \geq 0$, we select $t_{1} \geq t_{0}$ such that (A.2) holds. Let $e_{i} \in \mathbb{R}^{m_{1}}$ be a unit column vector with the $i$ th element equal to 1 . Take the initial pair $\left(t_{1}, Y_{t_{1}}\right)$ for (16) with $Y_{t_{1}}=e_{i}$. We have

$$
Y_{t+1}=\Psi_{t+1, t_{1}} e_{i}+\sum_{k=t_{1}}^{t} \Psi_{t+1, k+1} H_{k} V_{k}, \quad t \geq t_{1} .
$$

By mean square consensus there exists a random variable $\eta_{i}$ such that

$$
\lim _{t \rightarrow \infty} E\left|Y_{t+1}-\eta_{i} \mathbf{1}_{m_{1}}\right|^{2}=0
$$

We have

$$
\begin{aligned}
E\left|\Psi_{t+1, t_{1}} e_{i}-\eta_{i} \mathbf{1}_{m_{1}}\right|^{2} \leq & 2 E\left|Y_{t+1}-\eta_{i} \mathbf{1}_{m_{1}}\right|^{2} \\
& +2 E\left|\xi_{t_{1}, t-t_{1}+1}\right|^{2} \\
\leq & 2 E\left|Y_{t+1}-\eta_{i} \mathbf{1}_{m_{1}}\right|^{2}+\epsilon .
\end{aligned}
$$

This combined with (A.5) implies that there exists $t_{2} \geq t_{1}$ such that for $t \geq t_{2}$ and each $e_{i}, 1 \leq i \leq m_{1}, E \mid \Psi_{t+1, t_{1}} e_{i}-$ $\left.\eta_{i} \mathbf{1}_{m_{1}}\right|^{2} \leq 2 \epsilon$. Since $\Psi_{t+1, t_{1}} e_{i}$ is the $i$ th column of $\Psi_{t+1, t_{1}}$, it follows that

$$
E\left|\Psi_{t+1, t_{1}}-\mathbf{1}_{m_{1}}\left(\eta_{1}, \ldots, \eta_{m_{1}}\right)\right|^{2} \leq 2 m_{1} \epsilon, \quad t \geq t_{2} .
$$

Here we will not directly analyze the asymptotic property of $\Psi_{t+1, t_{1}}$ since $t_{1}$ changes with $\epsilon$. In fact $\left(\eta_{1}, \ldots, \eta_{m_{1}}\right)$ also changes with $t_{1}$ and $\epsilon$. For the fixed $t_{0}$, we have

$$
\begin{aligned}
& \left|\Psi_{t+1, t_{0}}-\mathbf{1}_{m_{1}}\left(\eta_{1}, \ldots, \eta_{m_{1}}\right) \Psi_{t_{1}, t_{0}}\right|^{2} \\
& \quad=\left|\Psi_{t+1, t_{1}} \Psi_{t_{1}, t_{0}}-\mathbf{1}_{m_{1}}\left(\eta_{1}, \ldots, \eta_{m_{1}}\right) \Psi_{t_{1}, t_{0}}\right|^{2} \\
& \quad \leq\left|\Psi_{t+1, t_{1}}-\mathbf{1}_{m_{1}}\left(\eta_{1}, \ldots, \eta_{m_{1}}\right)\right|^{2}\left|\Psi_{t_{1}, t_{0}}\right|^{2} \\
& \quad \leq m_{1}\left|\Psi_{t+1, t_{1}}-\mathbf{1}_{m_{1}}\left(\eta_{1}, \ldots, \eta_{m_{1}}\right)\right|^{2} .
\end{aligned}
$$

Hence,

$$
E\left|\Psi_{t+1, t_{0}}-\mathbf{1}_{m_{1}}\left(\eta_{1}, \ldots, \eta_{m_{1}}\right) \Psi_{t_{1}, t_{0}}\right|^{2} \leq 2 m_{1}^{2} \epsilon
$$

where $t \geq t_{2}$. Since $\epsilon>0$ is arbitrary, (A.6) implies that $\left\{\Psi_{t+1, t_{0}}, t \geq t_{0}\right\}$ is a Cauchy sequence in the $L_{2}$ norm and converges in mean square to a random matrix $\Pi_{t_{0}}$. Clearly, for each $\omega, \Pi_{t_{0}}(\omega)$ is a stochastic matrix. Since $\mathbf{1}_{m_{1}}\left(\eta_{1}, \ldots, \eta_{m_{1}}\right) \Psi_{t_{1}, t_{0}}$ in (A.6) is a matrix of identical rows, the mean square error between any two rows of $\Psi_{t+1, t_{0}}$ is at most $4 m_{1}^{2} \epsilon$ for all $t \geq$ $t_{2}$, which implies that $\Pi_{t_{0}}$ has identical rows since $\epsilon>0$ is arbitrary.

We proceed to show that the mean square convergence of $\Psi_{t+1, t_{0}}$ implies convergence w.p.1. Since $\lim _{t \rightarrow \infty} E \mid \Psi_{t+1, t_{0}}$ $\left.\Pi_{t_{0}}\right|^{2}=0$, there exists a sequence of integers $t_{0}<\tau_{1}<\tau_{2}<$ $\ldots$ such that $\left\{\Psi_{\tau_{k}, t_{0}}, k \geq 1\right\}$ converges to $\Pi_{t_{0}}$ for all $\omega \in \Omega \backslash N$, where $N$ is a null set.

For any $\omega \in \Omega \backslash N$ and $\epsilon>0$, there exists $k_{0}$ depending on $\omega$ such that for all $k \geq k_{0}$

$$
\left|\Psi_{\tau_{k}, t_{0}}(\omega)-\Pi_{t_{0}}(\omega)\right| \leq \epsilon .
$$


For any $t \geq \tau_{k_{0}}$, since $\Psi_{t, \tau_{k_{0}}}$ is a stochastic matrix and $\Pi_{t_{0}}$ has identical rows

$$
\begin{aligned}
\left|\Psi_{t, t_{0}}-\Pi_{t_{0}}\right| & =\left|\Psi_{t, \tau_{k_{0}}} \Psi_{\tau_{k_{0}}, t_{0}}-\Pi_{t_{0}}\right| \\
& =\left|\Psi_{t, \tau_{k_{0}}} \Psi_{\tau_{k_{0}}, t_{0}}-\Psi_{t, \tau_{k_{0}}} \Pi_{t_{0}}\right| \\
& \leq\left|\Psi_{t, \tau_{k_{0}}}\right|\left|\Psi_{\tau_{k_{0}}, t_{0}}-\Pi_{t_{0}}\right|
\end{aligned}
$$

Hence, for each $\omega \in \Omega \backslash N$ and $t \geq \tau_{k_{0}},\left|\Psi_{t, t_{0}}(\omega)-\Pi_{t_{0}}(\omega)\right| \leq$ $\sqrt{m_{1}} \epsilon$, which implies that $\Psi_{t, t_{0}}$ converges to $\Pi_{t_{0}}$ w.p.1. This completes the proof of necessity.

\section{APPENDIX B}

\section{PROOF OF THEOREM 6}

Let $\left\{e_{1}, \ldots, e_{k}\right\}$ denote the canonical basis of $\mathbb{R}^{k}$.

Lemma B.1: Let $\left\{\tilde{M}_{t}, t \geq 0\right\}$ be a sequence of $k \times k$ stochastic matrices. Then it has ergodic backward products if and only if

$$
Y_{t+1}=\tilde{M}_{t} Y_{t}
$$

achieves consensus (i.e., $\lim _{t \rightarrow \infty} Y_{t}=y^{*} \mathbf{1}_{k}$ for some $y^{*} \in \mathbb{R}$ ) for any given initial pair $\left(t_{0}, Y_{t_{0}}\right)$ where $Y_{t_{0}} \in\left\{e_{1}, \ldots e_{k}\right\}$, $t_{0} \geq 0$.

Proof: Necessity is obvious. We show sufficiency. For any $s \geq 0$, we run the linear system (B.1) by taking the initial pairs $\left(s, e_{j}\right), 1 \leq j \leq k$, respectively. Then by consensus

$$
\begin{aligned}
& \tilde{M}_{t} \ldots \tilde{M}_{s+1} \tilde{M}_{s}= \\
& \quad \tilde{M}_{t} \ldots \tilde{M}_{s+1} \tilde{M}_{s}\left(e_{1}, \ldots, e_{k}\right) \stackrel{t \rightarrow \infty}{\longrightarrow}\left(c_{1} \mathbf{1}_{k}, \ldots, c_{k} \mathbf{1}_{k}\right)
\end{aligned}
$$

for some constants $c_{1}, \ldots, c_{k}$ depending on $s$. So $\left\{\tilde{M}_{t}, t \geq 0\right\}$ has ergodic backward products.

Lemma B.2: Let $\left\{\tilde{M}_{t}, t \geq 0\right\}$ be $k \times k$ stochastic matrices with ergodic backward products, and

$$
Y_{t+1}=\tilde{M}_{t} Y_{t}+\xi_{t}, \quad t \geq 0
$$

where $\xi_{t} \in \mathbb{R}^{k}$ and $\sum_{t=0}^{\infty}\left|\xi_{t}\right|<\infty$. Then $\lim _{t \rightarrow \infty} Y_{t}=y^{*} \mathbf{1}_{k}$ for some $y^{*} \in \mathbb{R}$.

Proof: Denote $\Phi_{t, s}=\tilde{M}_{t-1} \ldots \tilde{M}_{s}$ for $t>s$, and $\Phi_{s, s}:=$ I. For $t>t_{0} \geq 0$

$$
Y_{t}=\Phi_{t, t_{0}} Y_{t_{0}}+\sum_{s=t_{0}}^{t-1} \Phi_{t, s+1} \xi_{s}
$$

Since $\left|\Phi_{s_{2}, s_{1}}\right| \leq \sqrt{k}$ for $s_{2} \geq s_{1}$, there exists a fixed $C$ such that $\sup _{t>0}\left|Y_{t}\right| \leq C$.

Given any $\varepsilon>0$, we may find a sufficiently large $t_{0}$ such that

$$
\sup _{t_{0}^{\prime \prime}>t_{0}^{\prime} \geq t_{0}}\left|\sum_{s=t_{0}^{\prime}}^{t_{0}^{\prime \prime}-1} \Phi_{t_{0}^{\prime \prime}, s+1} \xi_{s}\right| \leq \varepsilon .
$$

Denote $\Phi_{t, t_{0}} Y_{t_{0}}=\left[\eta_{t, 1}, \ldots, \eta_{t, k}\right]^{T}$ and $Y_{t}=\left[Y_{t}^{1}, \ldots, Y_{t}^{k}\right]^{T}$. For a sufficiently large $t_{1}>t_{0}$, by ergodicity we have $\max _{1 \leq i, j \leq k}\left|\eta_{t, i}-\eta_{t, j}\right| \leq \varepsilon$ for all $t \geq t_{1}$. Hence, $\max _{1 \leq i, j \leq k}\left|Y_{t}^{i}-Y_{t}^{j}\right| \leq 3 \varepsilon$ for all $t \geq t_{1}$. Since $\varepsilon$ is arbitrary

$$
\lim _{t \rightarrow \infty} \max _{1 \leq i, j \leq k}\left|Y_{t}^{i}-Y_{t}^{j}\right|=0
$$

Next, for $t^{\prime}>t \geq t_{1}$, we have

$$
\begin{aligned}
Y_{t^{\prime}} & =\Phi_{t^{\prime}, t} Y_{t}+\sum_{s=t}^{t^{\prime}-1} \Phi_{t^{\prime}, s+1} \xi_{s} \\
& =Y_{t}+\left(Y_{t}^{1} \mathbf{1}_{k}-Y_{t}\right)+\Phi_{t^{\prime}, t}\left(Y_{t}-Y_{t}^{1} \mathbf{1}_{k}\right)+\sum_{s=t}^{t^{\prime}-1} \Phi_{t^{\prime}, s+1} \xi_{s}
\end{aligned}
$$

By (B.3), (B.4), $\left\{Y_{t}, t \geq 0\right\}$ is a Cauchy sequence in $\mathbb{R}^{k}$. By using (B.4) again, there exists $y^{*}$ such that $\lim _{t \rightarrow \infty} Y_{t}=y^{*} \mathbf{1}_{k}$.

Lemma B.3: Consider (23) with the initial pair $\left(t_{0}, \mathbf{X}_{t_{0}}\right)$. Assume that $\left\{\mathbf{A}_{t}, t \geq 0\right\}$ is $\left(\delta_{t}\right)$-compatible with $\left\{\tilde{G}_{t}, t \geq 0\right\}$ such that (18), (19) hold for $t \geq t_{c}$. If $d^{*} \geq 1$, we have

$$
\max _{1 \leq d \leq d^{*}}\left|X_{t}-X_{t}^{(-d)}\right| \leq C \delta_{t}^{*}, \quad t \geq \max \left\{t_{0}, t_{c}\right\}+d^{*}
$$

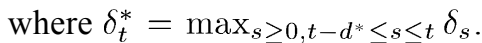

Proof: $\sup _{t>t_{0}}\left|\mathbf{X}_{t}\right| \leq C$ for some constant $C$. For $t \geq$ $\max \left\{t_{0}, t_{c}\right\}+d^{*}$ and any $1 \leq d \leq d^{*}$, we have $X_{t}^{(-d)}=X_{t-d}$ and $\left|A_{00, t-1}-I\right| \leq C \delta_{t-1}$. Here $C$ may take different values. Hence,

$$
\begin{aligned}
\left|X_{t}-X_{t-1}\right| & =\left|A_{00, t-1} X_{t-1}+\sum_{d=1}^{d^{*}} A_{0 d, t-1} X_{t-1}^{(-d)}-X_{t-1}\right| \\
& \leq C \delta_{t-1} .
\end{aligned}
$$

Similarly,

$$
\left|X_{t-l+1}-X_{t-l}\right| \leq C \delta_{t-l}, \quad 1 \leq l \leq d^{*} .
$$

Hence, for any $d \leq d^{*},\left|X_{t}-X_{t}^{(-d)}\right|=\left|X_{t}-X_{t-d}\right| \leq$ $C\left(\delta_{t-1}+\ldots+\delta_{t-d}\right) \leq C \delta_{t}^{*}$.

Proof of Theorem 6: Sufficiency-Suppose that $\left\{M_{t}^{A}, t \geq\right.$ $0\}$ has ergodic backward products. Consider (23) with the initial pair $\left(t_{0}, \mathbf{X}_{t_{0}}\right)$. For $t \geq t_{0}$

$$
\begin{aligned}
X_{t+1} & =A_{00, t} X_{t}+\sum_{d=1}^{d^{*}} A_{0 d, t} X_{t}^{(-d)} \\
& =M_{t}^{A} X_{t}+\sum_{d=1}^{d^{*}} A_{0 d, t}\left(X_{t}^{(-d)}-X_{t}\right) .
\end{aligned}
$$

Denote $\xi_{t}=\sum_{d=1}^{d^{*}} A_{0 d, t}\left(X_{t}^{(-d)}-X_{t}\right)$. Then by Lemma B.3, $\left|\xi_{t}\right| \leq C \delta_{t} \delta_{t}^{*} \leq C\left(\delta_{t}^{*}\right)^{2}$ for $t \geq \max \left\{t_{0}, t_{c}\right\}+d^{*}$. So $\sum_{t=t_{0}}^{\infty}\left|\xi_{t}\right|<\infty$. By Lemma B.2, $\lim _{t \rightarrow \infty} X_{t}=x_{\infty} 1_{n}$ for some $x_{\infty} \in \mathbb{R}$. Hence, $\lim _{t \rightarrow \infty} \mathbf{X}_{t}=x_{\infty} \mathbf{1}_{n\left(d^{*}+1\right)}$. By Lemma B.1, $\left\{\mathbf{A}_{t}, t \geq 0\right\}$ has ergodic backward products.

Necessity-Suppose that $\left\{\mathbf{A}_{t}, t \geq 0\right\}$ has ergodic backward products. By Proposition 5, $\left\{M_{t}^{A}, t \geq 0\right\}$ satisfies the $\left(\delta_{t}\right)$-compatibility condition with some constants $t_{c}, \underline{c}, \bar{c}$. Consider $X_{t+1}=M_{t}^{A} X_{t}$ with the initial pair $\left(t_{0}, X_{t_{0}}\right)$. Since (20) holds 
for $M_{t}^{A},\left|X_{t+1}-X_{t}\right| \leq C \delta_{t}$ for $t \geq \max \left\{t_{0}, t_{c}\right\}$ and some $C$. Define $X_{t+1}^{(-1)}=X_{t}, X_{t+1}^{(-d)}=X_{t}^{(-(d-1))}$ for $d=2, \ldots, d^{*}$. Fix any initial condition $\left(X_{t_{0}}, X_{t_{0}}^{(-1)}, \ldots, X_{t_{0}}^{\left(-d^{*}\right)}\right)$. By using the method in proving Lemma B.3, we may show that

$$
\max _{1 \leq d \leq d^{*}}\left|X_{t}-X_{t}^{(-d)}\right| \leq C \delta_{t}^{*}, \quad t \geq \max \left\{t_{0}, t_{c}\right\}+d^{*}
$$

Then

$$
X_{t+1}=A_{00, t} X_{t}+\sum_{d=1}^{d^{*}} A_{0 d, t} X_{t}^{(-d)}+\sum_{d=1}^{d^{*}} A_{0 d, t}\left(X_{t}-X_{t}^{(-d)}\right) .
$$

Letting $\mathbf{X}_{t}=\left[X_{t} ; X_{t}^{(-1)} ; \ldots ; X_{t}^{\left(-d^{*}\right)}\right]$, we may write

$$
\mathbf{X}_{t+1}=\mathbf{A}_{t} \mathbf{X}_{t}+\xi_{t}, \quad t \geq t_{0}
$$

where $\xi_{t}=\left[\sum_{d=1}^{d^{*}} A_{0 d, t}\left(X_{t}-X_{t}^{(-d)}\right) ; 0_{n d^{*} \times 1}\right]$. We have $\sum_{t=t_{0}}^{\infty}\left|\xi_{t}\right|<\infty$. Since $\left\{\mathbf{A}_{t}, t \geq 0\right\}$ has ergodic backward products, by Lemma B.2 there exists $x_{\infty}$ such that $\lim _{t \rightarrow \infty} \mathbf{X}_{t}=x_{\infty} \mathbf{1}_{n\left(d^{*}+1\right)}$, which implies $\lim _{t \rightarrow \infty} X_{t}=x_{\infty} \mathbf{1}_{n}$. Since $\left(t_{0}, X_{t_{0}}\right)$ is arbitrary, Lemma B.1 implies that $\left\{M_{t}^{A}, t \geq 0\right\}$ has ergodic backward products.

\section{APPENDIX C}

\section{ProOf OF THEOREM 8}

Lemma C.1: Let $t_{1} \geq 2, T \geq 0$, and $\gamma \in(0,1]$. Then $\sum_{t=t_{1}}^{t_{1}+T} t^{-\gamma} \leq(T+1) /\left(t_{1}-1\right)^{\gamma}$.

Proof: If $\gamma \in(0,1)$, we have

$$
\begin{aligned}
\sum_{t=t_{1}}^{t_{1}+T} t^{-\gamma} & \leq \int_{t_{1}-1}^{t_{1}+T} t^{-\gamma} d t \\
& =\frac{\left(t_{1}-1\right)^{1-\gamma}}{1-\gamma}\left\{\left[1+(T+1) /\left(t_{1}-1\right)\right]^{1-\gamma}-1\right\} \\
& \leq \frac{\left(t_{1}-1\right)^{1-\gamma}}{1-\gamma}(1-\gamma) \frac{T+1}{t_{1}-1}=\frac{T+1}{\left(t_{1}-1\right)^{\gamma}} .
\end{aligned}
$$

If $\gamma=1$, the estimate is similar and the detail is omitted.

Proof of Theorem 8: We say that condition (C1) is satisfied infinitely often (i.o.) by elements in a sequence $\left\{h_{t}, t \geq T_{0}\right\}$ if given any $T_{1} \geq T_{0}$, there exists $t \geq T_{1}$ such that $h_{t}$ satisfies (C1). It suffices to show $\lim _{t \rightarrow \infty} Z_{t}=c 1_{n}$. We consider the initial pair $\left(0, X_{0}\right)$. The proof with a general initial pair $\left(t_{0}, X_{t_{0}}\right)$ is similar. We prove by induction for the $n$ components of $Z_{t}$.

Step 1) By Proposition $7, \lim _{t \rightarrow \infty} z_{t}^{1}=z_{\infty}^{1}$ for some finite $z_{\infty}^{1}$

Step 2) Assume that for $l \in\{1, \ldots, n-1\}$,

$$
\lim _{t \rightarrow \infty} z_{t}^{k}=z_{\infty}^{1}
$$

for all $k \leq l$. Next we show that (C.1) holds for $k=l+1$, which is given in several sub-steps.

Step 2.1 (Contradiction argument) Suppose that (C.1) is not true for $k=l+1$, which implies that there exists $0<\epsilon_{0} \leq 1$ such that

$$
\left|z_{t}^{l+1}-z_{\infty}^{1}\right| \geq \epsilon_{0} \quad \text { i.o. }
$$

Since $\lim \sup _{t \rightarrow \infty} z_{t}^{l+1} \leq \lim _{t \rightarrow \infty} z_{t}^{1}=z_{\infty}^{1}$ by the ordering of the elements in $Z_{t}$, (C.2) implies that

$$
z_{t}^{l+1} \leq z_{\infty}^{1}-\epsilon_{0} \quad \text { i.o. }
$$

Step 2.2 (Estimate of the $(n-l)$ lowest trajectories) By compatibility, suppose that (20), (21) hold with $\delta_{t}=a_{t}, t \geq t_{c} \geq 0$. For any $\epsilon_{1} \in\left(0, \epsilon_{0} / 3\right)$, by the induction assumption (C.1), there exists $T\left(\epsilon_{1}\right)>t_{c}$ such that for all $t \geq T\left(\epsilon_{1}\right)$

$\left|z_{t}^{k}-z_{\infty}^{1}\right| \leq \epsilon_{1}, \quad k=1, \ldots, l$.

By (C.3), there exists $t_{1} \geq \max \left\{T\left(\epsilon_{1}\right), 2\right\}$ such that $z_{t_{1}}^{j} \leq z_{\infty}^{1}-\epsilon_{0}$ for $j=l+1, \ldots, n$ since $z_{t_{1}}^{l+1} \geq z_{t_{1}}^{l+2} \geq \ldots \geq z_{t_{1}}^{n}$. Consider the vector $Z_{t_{1}}$ and use our convention of associating a component with a node. Suppose

$z_{t_{1}}^{1}=x_{t_{1}}^{i_{1}}, z_{t_{1}}^{2}=x_{t_{1}}^{i_{2}}, \ldots, z_{t_{1}}^{l}=x_{t_{1}}^{i_{l}}$

for $l \geq 1$ distinct elements $i_{1}, \ldots, i_{l}$ in $\mathcal{N}=\{1, \ldots, n\}$, which implicitly depend on $t_{1}$. Denote $S_{t_{1}}=\left\{i_{1}, \ldots, i_{l}\right\}$ and its complement $S_{t_{1}}^{c}=\mathcal{N} \backslash S_{t_{1}}$. Note that $\sup _{t \geq 0,1 \leq k \leq n}\left|x_{t}^{k}\right| \leq C_{X_{0}}:=\max _{1 \leq i \leq n}\left|x_{0}^{i}\right|$. Denote $\bar{M}_{t}=\left(a_{i j}(t)\right)_{1 \leq i, j \leq n}$. For $j \in S_{t_{1}}^{c}$ and $t \geq t_{1}$, it follows from (20) with the substitution of $\delta_{t}=a_{t}$ that

$$
\begin{aligned}
x_{t+1}^{j} & =\sum_{k=1}^{n} a_{j k}(t) x_{t}^{k} \\
& =\sum_{k \in S_{t_{1}}^{c}} a_{j k}(t) x_{t}^{k}+\sum_{k \in S_{t_{1}}} a_{j k}(t) x_{t}^{k} \\
& \leq \max _{k \in S_{t_{1}}^{c}} x_{t}^{k}+c_{1} / t^{\gamma}
\end{aligned}
$$

where $c_{1}$ depends only on $\left\{M_{t}, t \geq 0\right\}$ and $C_{X_{0}}$. Since $j \in S_{t_{1}}^{c}$ is arbitrary, (C.6) implies that

$\max _{k \in S_{t_{1}}^{c}} x_{t+1}^{k} \leq \max _{k \in S_{t_{1}}^{c}} x_{t}^{k}+c_{1} / t^{\gamma}, \quad t \geq t_{1}$.

Subsequently, we choose a large $T$ such that

$$
\sum_{t=t_{1}}^{t_{1}+T} c_{1} / t^{\gamma} \leq \epsilon_{0} / 3
$$

By Lemma C.1, we take

$$
T=\left\lfloor\epsilon_{0}\left(t_{1}-1\right)^{\gamma} /\left(3 c_{1}\right)-1\right\rfloor
$$

to ensure (C.8). We use $\lfloor r\rfloor$ to denote the greatest integer bounded from above by $r$. Without loss of generality, assume that given 
$\left(\epsilon_{0}, \epsilon_{1}\right)$, a sufficiently large $t_{1}$ has been selected such that

$$
\frac{\epsilon_{0}\left(t_{1}-1\right)^{\gamma}}{3 c_{1}} \geq 4
$$

The iteration of (C.7) yields

$$
\begin{aligned}
\max _{t_{1} \leq t \leq t_{1}+T+1} \max _{k \in S_{t_{1}}^{c}} x_{t}^{k} \leq & \max _{k \in s_{t_{1}}^{c}} x_{t_{1}}^{k}+ \\
& c_{1} \sum_{t=t_{1}}^{t_{1}+T} 1 / t^{\gamma} \\
& \leq z_{t_{1}}^{l+1}+\epsilon_{0} / 3 \leq z_{\infty}^{1}-\epsilon_{0}+\epsilon_{0} / 3 \\
& =z_{\infty}^{1}-\left(2 \epsilon_{0}\right) / 3 .
\end{aligned}
$$

By (C.4) and (C.11), the states of nodes $i_{1}, \ldots, i_{l}$ remain to be the first $l$ greatest states for $t_{1} \leq t \leq t_{1}+T+1$ although the position of these states may change with time when listed in $Z_{t}$. Now by $\epsilon_{1}<\epsilon_{0} / 3$, it follows from (C.4) and (C.11) that for any $i \in S_{t_{1}}$ and any $j \in S_{t_{1}}^{c}$

$\left|x_{t}^{i}-z_{\infty}^{1}\right| \leq \epsilon_{1}, \quad x_{t}^{j} \leq x_{t}^{i}-\epsilon_{0} / 3$

where $t_{1} \leq t \leq t_{1}+T+1$ and $T$ is defined by (C.9).

Step 2.3 (Estimate of the $l$ highest trajectories) For node $i \in S_{t_{1}}$

$$
\begin{aligned}
x_{t+1}^{i}= & \sum_{k \in S_{t_{1}}} a_{i k}(t) x_{t}^{k}+\sum_{k \in S_{t_{1}}^{c}} a_{i k}(t) x_{t}^{k} \\
= & x_{t}^{i}+\left[a_{i i}(t)-1\right] x_{t}^{i}+\sum_{k \in S_{t_{1}} \backslash\{i\}} a_{i k}(t) x_{t}^{k} \\
& +\sum_{k \in S_{t_{1}}^{c}} a_{i k}(t) x_{t}^{k} \\
= & x_{t}^{i}+\sum_{k \in S_{t_{1}} \backslash\{i\}} a_{i k}(t)\left(x_{t}^{k}-x_{t}^{i}\right) \\
& +\sum_{k \in S_{t_{1}}^{c}} a_{i k}(t)\left(x_{t}^{k}-x_{t}^{i}\right)
\end{aligned}
$$

where $t_{1} \leq t \leq t_{1}+T$. It follows from (C.12) that

$$
\begin{aligned}
\left|\sum_{k \in S_{t_{1}} \backslash\{i\}} a_{i k}(t)\left(x_{t}^{k}-x_{t}^{i}\right)\right| & \leq 2 \epsilon_{1} \sum_{k \in S_{t_{1}} \backslash\{i\}} a_{i k}(t) \\
& \leq\left(2 \epsilon_{1}\right) c_{2} t^{-\gamma}
\end{aligned}
$$

where $c_{2}$ depends only on $\{M(t), t \geq 0\}$. We have

$$
\sum_{k \in S_{t_{1}}^{c}} a_{i k}(t)\left(x_{t}^{k}-x_{t}^{i}\right) \leq \sum_{k \in S_{t_{1}}^{c}} a_{i k}(t)\left(-\epsilon_{0} / 3\right)
$$

Denote $g_{t}=\sum_{i \in S_{t_{1}}} x_{t}^{i}$. Hence, by (C.13),

$g_{t+1} \leq g_{t}+2 \epsilon_{1} c_{2} n t^{-\gamma}-\left(\frac{\epsilon_{0}}{3}\right) \sum_{i \in S_{t_{1}}} \sum_{k \in S_{t_{1}}^{c}} a_{i k}(t)$.

Since $\tilde{G}_{t}$ is strongly connected, there exists a pair $(i, k) \in S_{t_{1}} \times S_{t_{1}}^{c}$ such that $(k, i)$ is an edge of $\tilde{G}_{t}$ and $a_{i k}(t) \geq c_{3} t^{-\gamma}$ for $t \geq t_{c}$ by compatibility, where $c_{3}>0$ is determined by $\left\{M_{t}, t \geq 0\right\}$.

Subsequently, it follows that

$g_{t+1} \leq g_{t}+2 \epsilon_{1} c_{2} n t^{-\gamma}-\left(\frac{\epsilon_{0}}{3}\right) c_{3} t^{-\gamma}$

where $t_{1} \leq t \leq t_{1}+T$. Since $c_{2}$ and $c_{3}$ depend only on $\{M(t), t \geq 0\}$, we may further assume that a sufficient small $\epsilon_{1}$ has been selected such that

$$
2 \epsilon_{1} c_{2} n<\frac{\epsilon_{0} c_{3}}{6} .
$$

Iterating (C.15), we have

$$
g_{t_{1}+T+1} \leq g_{t_{1}}-\sum_{t=t_{1}}^{t_{1}+T}\left(\frac{\epsilon_{0} c_{3}}{6}\right) t^{-\gamma} .
$$

Step 2.4 (A contradiction) Since $t_{1} \geq 2$ and (C.10) holds, for $T$ given by (C.9), it follows that

$$
\begin{aligned}
T & \geq \epsilon_{0}\left(t_{1}-1\right)^{\gamma} /\left(3 c_{1}\right)-2 \\
& \geq \epsilon_{0}\left(t_{1}-1\right)^{\gamma} /\left(6 c_{1}\right) \geq \epsilon_{0} t_{1}^{\gamma} /\left(12 c_{1}\right) .
\end{aligned}
$$

Denote $\eta=\epsilon_{0} /\left(12 c_{1}\right)$. Then $\eta \leq 1 /\left(12 c_{1}\right)$ since $\epsilon_{0} \leq 1$.

(a) If $0<\gamma<1$

$$
\begin{aligned}
\sum_{t=t_{1}}^{t_{1}+T} t^{-\gamma} & \geq \int_{t_{1}}^{t_{1}+T} v^{-\gamma} d v \geq \int_{t_{1}}^{t_{1}+\eta t_{1}^{\gamma}} v^{-\gamma} d v \\
& =t_{1}^{1-\gamma}\left[\left(1+\eta t_{1}^{\gamma-1}\right)^{1-\gamma}-1\right] /(1-\gamma) .
\end{aligned}
$$

For $f(v)=(1+v)^{1-\gamma}, v \in\left[0,1 /\left(12 c_{1}\right)\right]$, there exists a constant $D_{1}>0$ depending only on $\gamma$ and $c_{1}>0$ such that $f(v) \geq 1+D_{1} v$ for all $v \in\left[0,1 /\left(12 c_{1}\right)\right]$. Now $\eta t_{1}^{\gamma-1} \leq 1 /\left(12 c_{1}\right)$ and

$$
\begin{aligned}
\sum_{t=t_{1}}^{t_{1}+T} t^{-\gamma} & \geq t_{1}^{1-\gamma}\left(1+D_{1} \eta t_{1}^{\gamma-1}-1\right) /(1-\gamma) \\
& =D_{1} \epsilon_{0} /\left[12 c_{1}(1-\gamma)\right] .
\end{aligned}
$$

So for $0<\gamma<1$, we obtain

$$
g_{t_{1}+T+1} \leq g_{t_{1}}-D_{1} \epsilon_{0}^{2} c_{3} /\left[72 c_{1}(1-\gamma)\right] .
$$


On the other hand, by (C.12) we have

$$
\left|g_{t_{1}+T+1}-g_{t_{1}}\right| \leq 2 n \epsilon_{1} .
$$

If we take

$$
0<\epsilon_{1}<\min \left\{\frac{\epsilon_{0}}{3}, \epsilon_{0} c_{3} /\left(12 n c_{2}\right), D_{1} \epsilon_{0}^{2} c_{3} /\left[144 n c_{1}(1-\gamma)\right]\right\}
$$

then (C.16), (C.17) lead to a contradiction. Hence, (C.1) holds for $k=l+1$.

(b) If $\gamma=1$, a similar argument may be used to show (C.1) for $k=l+1$ and the detail is omitted.

Step 3) Finally, we conclude that (C.1) holds for all $k \in \mathcal{N}$. This completes the proof.

\section{APPENDIX D}

Lemma D.1: Assume condition i) in Theorem 11 holds. Let $h \geq 1$ be a fixed integer and denote $\tilde{G}_{[t, t+s)}=\left(\mathcal{N}, \tilde{\mathcal{E}}_{[t, t+s)}\right)$ for $t \geq 0$ and $1 \leq s \leq h$. Then there exist constants $t_{c}^{\prime} \geq 1$ and $0<\underline{c}^{\prime} \leq \bar{c}^{\prime}$, all independent of $(t, s)$, such that for all $t \geq t_{c}^{\prime}$, $M^{t, s}:=M_{t+s-1} \ldots M_{t}$ satisfies

$$
\begin{array}{ll}
M^{t, s}(i, j) \leq \bar{c}^{\prime} t^{-\gamma}, & \forall 1 \leq i, j \leq n, \quad j \neq i \\
M^{t, s}(i, j) \geq \underline{c}^{\prime} t^{-\gamma}, & \forall(j, i) \in \tilde{\mathcal{E}}_{[t, t+s)}
\end{array}
$$

where $M^{t, s}(i, j)$ is the $(i, j)$ th element of $M^{t, s}$.

Proof: If $s=1$, the lemma is obvious. Below we consider $s \geq 2$ and $t \geq t_{c}$, where $t_{c}$ is selected such that (20), (21) hold for $t \geq t_{c}$ with constants $t_{c}, 0<\underline{c} \leq \bar{c}$.

For $j \neq i$, we have

$$
\begin{aligned}
& M^{t, s}(i, j) \\
& =\sum_{k_{1}, \ldots, k_{s-1}} M_{t+s-1}\left(i, k_{s-1}\right) \ldots M_{t+1}\left(k_{2}, k_{1}\right) M_{t}\left(k_{1}, j\right)
\end{aligned}
$$

where each $k_{l} \in\{1, \ldots, n\}$. Each factor of $M_{t+s-1}\left(i, k_{s-1}\right) \ldots M_{t+1}\left(k_{2}, k_{1}\right) M_{t}\left(k_{1}, j\right)$ is between 0 and 1 . Since $j \neq i$, there is at least one factor of the form $M_{t+r}(i, k)$ with $k \neq i$ and $0 \leq r \leq s-1$, so that it is bounded from above by $\bar{c} a_{t+r}$ by condition i). In addition, the number of terms in the summation has a finite upper bound depending only on $h$. Hence (D.1) follows.

We proceed to prove (D.2). Assume that $(j, i) \in \tilde{G}_{[t, t+s)}$. So there exists $0 \leq \xi \leq s-1$ such that $(j, i) \in \tilde{G}_{t+\xi}$. Consequently, by the compatibility condition $M_{t+\xi}(i, j) \geq \underline{c} a_{t+\xi}$. We have

$$
\begin{aligned}
M^{t, s} & (i, j) \\
= & \sum_{k_{1}, \ldots, k_{s-1}} M_{t+s-1}\left(i, k_{s-1}\right) \ldots M_{t+1}\left(k_{2}, k_{1}\right) M_{t}\left(k_{1}, j\right) \\
\geq & M_{t+s-1}(i, i) \ldots M_{t+\xi+1}(i, i) M_{t+\xi}(i, j) M_{t+\xi-1}(j, j) \\
& \ldots M_{t}(j, j) .
\end{aligned}
$$

If $\xi=0$ (resp., $\xi=s-1), M_{t+\xi}(i, j)$ takes the position of the most right (resp., left) term in (D.3).
By (20), there exist $t_{c}^{\prime} \geq t_{c}$ and $\delta>0$ such that $\min _{1 \leq i \leq n} M_{t+r}(i, i) \geq 1-\delta a_{t+r} \geq 1 / 2$ for $t \geq t_{c}^{\prime}$ and $r \geq 0$. We obtain

$$
\begin{aligned}
M^{t, s}(i, j) \geq\left(1-\delta a_{t+s-1}\right) \ldots & \left(1-\delta a_{t+\xi+1}\right) \underline{c} a_{t+\xi} \\
& \times\left(1-\delta a_{t+\xi-1}\right) \ldots\left(1-\delta a_{t}\right) .
\end{aligned}
$$

Since $0 \leq \xi<s \leq h$ for the fixed $h$, by (A1) we may select $t_{c}^{\prime}$ such that (D.2) holds.

Lemma D.2: Assume conditions i)-ii) in Theorem 11 holds. Then $\left\{\widehat{M}_{t}, t \geq 0\right\}$ is $\left(a_{t}\right)$-compatible with $\left\{\widehat{G}_{t}, t \geq 0\right\}$, where $\widehat{M}_{t}=M_{\tau_{t+1}-1} \ldots M_{\tau_{t}}$ and $\widehat{G}_{t}=\tilde{G}_{\left[\tau_{t}, \tau_{t+1}\right)}$.

Proof: There exists a fixed constant $h>0$ such that

$$
1 \leq \tau_{t+1}-\tau_{t} \leq h, \quad t \leq \tau_{t} \leq h t, \quad t \geq 0 .
$$

So the lemma follows from Lemma D.1.

\section{ACKNOWLEDGMENT}

The author would like to thank the anonymous reviewers and the Associate Editor Prof. C. Szepesvári for very useful comments and suggestions which have helped improve the presentation of the paper.

\section{REFERENCES}

[1] J. Abounadi, D. P. Bertsekas, and V. Borkar, "Stochastic approximation for nonexpansive maps: Application to Q-learning algorithms," SIAM J. Control Optim., vol. 41, no. 1, pp. 1-22, 2002.

[2] D. Acemoglu, A. Nedić, and A. Ozdaglar, "Convergence of rule-ofthumb learning rules in social networks," in Proc. 47th IEEE CDC, Cancun, Mexico, Dec. 2008, pp. 1714-1720.

[3] T. C. Aysal and K. E. Barner, "Convergence of consensus models with stochastic disturbances," IEEE Trans. Inf. Theory, vol. 56, no. 8, pp. 4101-4113, Aug. 2010.

[4] T. C. Aysal, M. J. Coates, and M. G. Rabbat, "Distributed average consensus with dithered quantization," IEEE Trans. Signal Process., vol. 56, no. 10, pp. 4905-4918, Oct. 2008.

[5] F. Bćnćzit, V. Blondel, P. Thiran, J. Tsitsiklis, and M. Vetterli, "Weighted gossip: Distributed averaging using non-doubly stochastic matrices," in Proc. Int. Symp. Inf. Theory, Austin, TX, Jun. 2010, pp. $1753-1757$.

[6] V. D. Blondel, J. M. Hendrickx, A. Olshevsky, and J. N. Tsitsiklis, "Convergence in multiagent coordination, consensus, and flocking," in Proc. 44th IEEE CDC-ECC'05, Seville, Spain, Dec. 2005, pp. 2996-3001.

[7] V. S. Bokar, "Asynchronous stochastic approximation," SIAM J. Control Optim., vol. 36, no. 3, pp. 840-851, 1998, Erratum: vol. 38, no. 2, pp. 662-663, 2000.

[8] S. Boyd, A. Ghosh, B. Prabhakar, and D. Shah, "Randomized gossip algorithms," IEEE Trans. Inform. Theory, vol. 52, no. 6, pp. 2508-2530, Jun. 2006.

[9] P. Brćmaud, Markov Chains: Gibbs Fields, Monte Carlo Simulation, and Queues. New York: Springer-Verlag, 1999.

[10] R. Carli, F. Fagnani, P. Frasca, T. Taylor, and S. Zampieri, "Average consensus on networks with transmission noise or quantization," in Proc. Eur. Control Conf., Kos, Greece, Jul. 2007, pp. 1852-1857.

[11] R. Carli, F. Fagnani, A. Speranzon, and S. Zampieri, "Communication constraints in the average consensus problem," Automatica, vol. 44, pp. 671-684, 2008.

[12] F. Cucker and E. Mordecki, "Flocking in noisy environments," J. Math. Pures Appl., vol. 89, no. 3, pp. 278-296, 2008.

[13] L. Fang and P. J. Antsaklis, "Asynchronous consensus protocols using nonlinear paracontraction theory," IEEE Trans. Autom. Control, vol. 53, no. 10, pp. 2351-2355, Nov. 2008.

[14] R. Gharavi and V. Anantharam, "Structure theorems for partially asynchronous iterations of a nonnegative matrix with random delays," Sādhanā, vol. 24, no. 4-5, pp. 369-423, 1999.

[15] B. Gharesifard and J. Cortćs, "Distributed strategies for generating weight-balanced and doubly stochastic digraphs," Eur. J. Control, 2012, to be published. 
[16] Y. Hatano and M. Mesbahi, "Agreement in random networks," IEEE Trans. Autom. Control, vol. 51, no. 11, pp. 1867-1872, Nov. 2005.

[17] M. Huang, S. Dey, G. N. Nair, and J. H. Manton, "Stochastic consensus over noisy networks with Markovian and arbitrary switches," Automatica, vol. 46, no. 10, pp. 1571-1583, 2010.

[18] M. Huang and J. H. Manton, "Coordination and consensus of networked agents with noisy measurements: Stochastic algorithms and asymptotic behavior," SIAM J. Control Optim., vol. 48, no. 1, pp. 134-161, 2009.

[19] M. Huang and J. H. Manton, "Stochastic consensus seeking with noisy and directed inter-agent communication: Fixed and randomly varying topologies," IEEE Trans. Autom. Control, vol. 55, no. 1, pp. 235-241, Jan. 2010.

[20] A. Jadbabaie, J. Lin, and A. S. Morse, "Coordination of groups of mobile autonomous agents using nearest neighbor rules," IEEE Trans. Autom. Control, vol. 48, no. 6, pp. 988-1000, Jun. 2003.

[21] S. Kar and J. M. F. Moura, "Distributed consensus algorithms in sensor networks with imperfect communication: Link failures and channel noise," IEEE Trans. Signal Process., vol. 57, no. 1, pp. 355-369, Jan. 2009.

[22] A. Kashyap, T. Başar, and R. Srikant, "Quantized consensus," Automatica, vol. 43, no. 7, pp. 1192-1203, 2007.

[23] V. Krishnamurthy, K. Topley, and G. Yin, "Consensus formation in a two-time-scale Markovian system," SIAM J. Multiscale Model. Simul., vol. 7, no. 4, pp. 1898-1927, 2009.

[24] H. J. Kushner and G. Yin, "Stochastic approximation algorithms for parallel and distributed processing," Stochastics, vol. 22, no. 3, pp. 219-250, 1987.

[25] I. K. L. Elsner and M. Neumann, "On the convergence of asynchronous paracontractions with applications to tomographic reconstruction from incomplete data," Linear Algebra and Appl., vol. 130, pp. 65-82, 1990.

[26] I. K. L. Elsner and M. Neumann, "Convergence of sequential and asynchronous nonlinear paracontractions," Numer. Math., vol. 62, pp. 305-319, 1992.

[27] T. Li and J.-F. Zhang, "Mean square average-consensus under measurement noises and fixed topologies," Automatica, vol. 45, no. 8, pp. 1929-1936, 2009.

[28] T. Li and J.-F. Zhang, "Consensus conditions of multi-agent systems with time-varying topologies and stochastic communication noises," IEEE Trans. Autom. Control, vol. 55, no. 9, pp. 2043-2057, Sep. 2010

[29] I. Matei, N. Martins, and J. S. Baras, "Almost sure convergence to consensus in Markovian random graphs," in Proc. 47th IEEE CDC, Cancun, Mexico, Dec. 2008, pp. 3535-3540.

[30] M. Nourian, P. E. Caines, R. P. Malhamć, and M. Huang, "Derivation of consensus algorithm dynamics from mean field stochastic control NCE equations," in Proc. 1st IFAC Workshop Estimation and Control of Netw. Syst., Venice, Italy, Sep. 2009.

[31] R. Olfati-Saber, J. A. Fax, and R. M. Murray, "Consensus and cooperation in networked multi-agent systems," Proc. IEEE, vol. 95, no. 1, pp. 215-233, Jan. 2007.

[32] R. Olfati-Saber and R. M. Murray, "Consensus problems in networks of agents with switching topology and time-delays," IEEE Trans. Autom. Control, vol. 49, no. 9, pp. 1520-1533, Sep. 2004.

[33] A. Olshevsky and J. N. Tsitsiklis, "On the nonexistence of quadratic Lyapunov functions for consensus algorithms," IEEE Trans. Autom. Control, vol. 53, no. 11, pp. 2642-2645, Dec. 2008.

[34] S. Patterson, B. Bamieh, and A. El Abbadi, "Convergence rates of distributed average consensus with stochastic link failures," IEEE Trans. Autom. Control, vol. 55, no. 4, pp. 880-892, Apr. 2010.
[35] R. Rajagopal and M. J. Wainwright, Network-based consensus averaging with general noisy channels, 2008 [Online]. Available: http:// arxiv.org/abs $/ 0805.0438$

[36] W. Ren and R. W. Beard, "Consensus seeking in multiagent systems under dynamically changing interaction topologies," IEEE Trans. Autom. Control, vol. 50, no. 5, pp. 655-661, May 2005.

[37] W. Ren, R. W. Beard, and E. M. Atkins, "A survey of consensus problems in multi-agent coordination," in Proc. Amer. Control Conf., Portland, OR, Jun. 2005, pp. 1859-1864.

[38] W. Ren, R. W. Beard, and D. B. Kingston, "Multi-agent Kalman consensus with relative uncertainty," in Proc. Amer. Control Conf., Portland, OR, Jun. 2005, pp. 1865-1870.

[39] I. D. Schizas, A. Ribeiro, and G. B. Giannakis, "Consensus in ad hoc WSNs with noisy links-part I: Distributed estimation of deterministic signals," IEEE Trans. Signal Process., vol. 56, no. 1, pp. 350-364, Jan. 2008.

[40] E. Seneta, Non-negative Matrices and Markov Chains, 2nd ed. New York: Springer, 2006, (revised printing).

[41] S. S. Stankovic, M. S. Stankovic, and D. M. Stipanovic, "Decentralized parameter estimation by consensus based stochastic approximation," in Proc. 46th IEEE CDC, New Orleans, LA, Dec. 2007, pp. 1535-1540.

[42] B. Touri and A. Nedić, "Distributed consensus over network with noisy links," in Proc. 12th Int. Conf. Info. Fusion, Seattle, WA, Jul. 2009, pp. $146-154$.

[43] J. N. Tsitsiklis, "Asynchronous stochastic approximation and Q-learning," Mach. Learn., vol. 16, pp. 185-202, 1994.

[44] J. N. Tsitsiklis, D. P. Bertsekas, and M. Athans, "Distributed asynchronous deterministic and stochastic gradient optimization algorithms," IEEE Trans. Autom. Control, vol. 31, no. 9, pp. 803-812, Sep. 1986

[45] J. Wolfowitz, "Products of indecomposable, aperiodic, stochastic matrices," Proc. American Math. Soc., vol. 14, no. 5, pp. 733-737, 1969

[46] L. Xiao, S. Boyd, and S.-J. Kim, "Distributed average consensus with least-mean-square deviation," J. Parallel Distrib. Comput., vol. 67, pp. 33-46, 2007.

[47] G. F. Young, L. Scardovi, and N. E. Leonard, "Robustness of noisy consensus dynamics with directed communication," in Proc. Amer. Control Conf., Baltimore, MD, Jun. 2010, pp. 6312-6317.

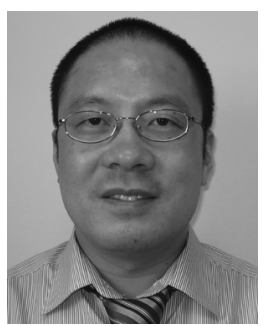

Minyi Huang (M'03) received the B.Sc. degree from Shandong University, Jinan, Shandong, China, in 1995, the M.Sc. degree from the Institute of Systems Science, Chinese Academy of Sciences, Beijing, China, in 1998, and the Ph.D. degree from the Department of Electrical and Computer Engineering, McGill University, Montreal, QC, Canada, in 2003, all in the area of systems and control.

He was a Research Fellow first in the Department of Electrical and Electronic Engineering, the University of Melbourne, Australia, from February 2004 to March 2006, and then in the Department of Information Engineering, Research School of Information Sciences and Engineering, the Australian National University, Canberra, Australia, from April 2006 to June 2007. He joined the School of Mathematics and Statistics, Carleton University, Ottawa, ON, Canada, as an Assistant Professor in July 2007, where he has been an Associate Professor since July 2011. His research interests include mean field stochastic control and dynamic games, multi-agent control and computation in distributed networks with applications. 